Article

\title{
Design, Synthesis, and Biological Activities of Novel 1,3,5-Trimethylpyrazole-Containing Malonamide Derivatives
}

\author{
Qi-Bo Li ${ }^{1}$, Min Liao ${ }^{1}$, Qing Liu ${ }^{1}$, Tong Feng ${ }^{1}$, Zhi-Yuan $\mathrm{Xu}^{1}$, Chang-Hui Rui ${ }^{2, *}$ and \\ Shang-Zhong Liu $1, *$ (i)
}

1 Department of Applied Chemistry, College of Science, China Agricultural University, Beijing 100193, China; lqb@cau.edu.cn (Q.-B.L.); cygnus@cau.edu.cn (M.L.); qingqing@cau.edu.cn (Q.L.); fibber@cau.edu.cn (T.F.); xuzhiyuan@cau.edu.cn (Z.-Y.X.)

2 Key Laboratory of Integrated Pest Management in Crops, Ministry of Agriculture, Institute of Plant Protection, Chinese Academy of Agricultural Sciences, Beijing 100193, China

* Correspondence: huirch@163.com (C.-H.R.); shangzho@cau.edu.cn (S.-Z.L.); Tel.: +86-10-62815944 (C.-H.R.); +86-10-62731070 (S.-Z.L.)

Received: 3 December 2018; Accepted: 30 January 2019; Published: 3 February 2019

\begin{abstract}
New 1,3,5-trimethylpyrazole-containing malonamide derivatives based on pyflubumide were designed, synthesized, and characterized using ${ }^{1} \mathrm{H}-\mathrm{NMR},{ }^{13} \mathrm{C}-\mathrm{NMR}$, and high-resolution mass spectra (HRMS). The results of preliminary bioassays showed that the target compounds possessed good activities against Tetranychus cinnabarinus, Plutella xylostella, and Aphis craccivora. Most of the target compounds exhibited moderate to good acaricidal activity against Tetranychus cinnabarinus at a concentration of $400 \mu \mathrm{g} / \mathrm{mL}$, and some showed moderate activity at a concentration of $200 \mu \mathrm{g} / \mathrm{mL}$; in particular, compounds $8 \mathrm{~m}$ and $8 \mathrm{p}$ exhibited $70.0 \%$ mortality. In addition, some of the target compounds exhibited good insecticidal activities against Plutella xylostella at a concentration of $200 \mu \mathrm{g} / \mathrm{mL}$, especially compounds $\mathbf{8 i}$ and $\mathbf{8 0}$, which achieved $100.0 \%$ mortality at a concentration of $100 \mu \mathrm{g} / \mathrm{mL}$. Interestingly, some of the target compounds exhibited potent anti-aphid activity against Aphis craccivora at a concentration of $200 \mu \mathrm{g} / \mathrm{mL}$; furthermore, compounds $\mathbf{8 p}$ and $\mathbf{8 q}$ demonstrated $100.0 \%$ anti-aphid activity at a concentration of $50 \mu \mathrm{g} / \mathrm{mL}$. The preliminary analyses of the structure-activity relationships (SAR) indicated that the acaricidal and insecticidal activities varied significantly depending on the type of substituent and substitution pattern, which provides guidance for the further investigation of such structural modifications.
\end{abstract}

Keywords: malonamide; pyflubumide; synthesis; acaricidal and insecticidal activities

\section{Introduction}

Amide bonds are an important functional group in many of the active ingredients of agrochemicals, such as the representative amide herbicides acetochlor and metolachlor [1,2] and the succinate dehydrogenase inhibitor (SDHI) fungicides boscalid and thifluzamide [3-5]. The nitrogen-containing heterocycle pyrazole ring is important in the field of medicinal chemistry [6-8], and also endows many pesticide molecules with versatile biological activities, as exemplified by fipronil, tebufenpyrad, pyraclostrobin, and pyroxasulfone [9-13].

Due to the discovery of the excellent performance of flubendiamide A (Figure 1), chlorantraniliprole B (Figure 1), and pyflubumide (Figure 2) against pest insects and phytophagous mites in crop protection and their subsequent commercialization, the design and synthesis of new compounds containing diamide groups has become a major trend in the search for potential compounds with insecticidal and acaricidal activities [14,15]. 
<smiles>Cc1cc(C(F)(F)C(F)(F)F)ccc1NC(=O)c1cccc(I)c1C(=O)NC(C)(C)CS(C)(=O)(O)O</smiles>

Flubendiamide $(\mathrm{A})$<smiles>CNC(=O)c1cc(Cl)cc(C)c1NC(=O)c1cc(Br)nn1-c1ncccc1Cl</smiles>

Chlorantraniliprole (B)

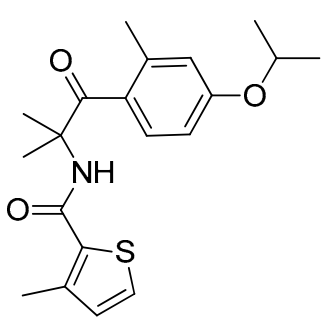

Isofetamid (C)

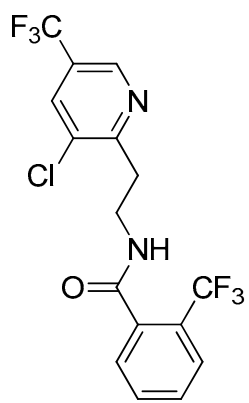

Fluopyram (D)

Figure 1. Structures of flubendiamide (A), chlorantraniliprole (B), isofetamid (C), and fluopyram (D).

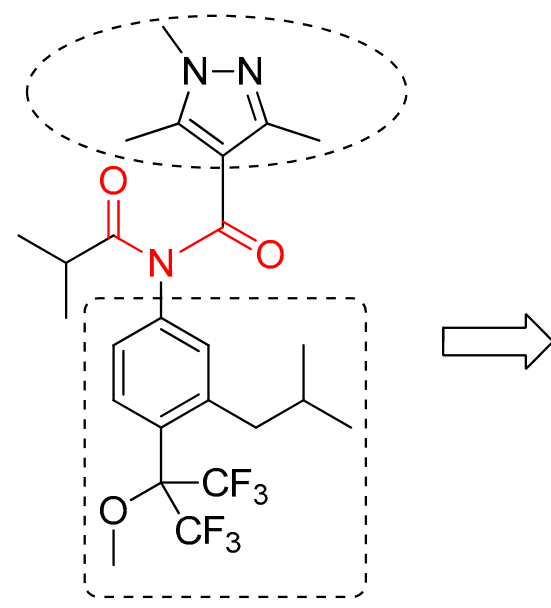

Pyflubumide

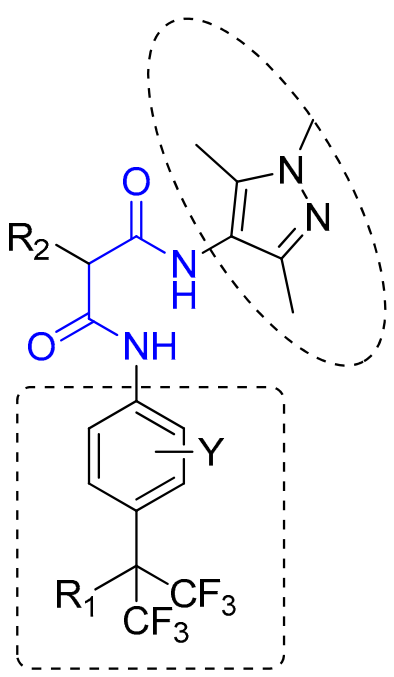

Target compounds

Figure 2. The design of the target compounds.

Pyflubumide is a novel acraricide with a unique carboxanilide structure and was specifically and highly active against all stages of agriculturally important spider mites, such as two-spotted spider mite, kanzawa spider mite. and citrus red mite $\left(\mathrm{LC}_{50}=1-3 \mu \mathrm{g} / \mathrm{mL}\right)$. However, it showed low activity against other pests, including hemiptera and lepidoptera $\left(\mathrm{LC}_{50}>500 \mu \mathrm{g} / \mathrm{mL}\right)$. Furthermore, it is harmless to non-target arthropods, beneficial insects, and natural enemies $[16,17]$. It was discovered by Nihon Nohyaku Co. Ltd. when a heptafluoroisopropyl group was added to a benzene ring during the optimization of carboxamide derivatives to discover new SDHI fungicidal molecules. As SDHI fungicides, pyflubumide acts on mitochondrial complex II in the respiratory chain of phytophagous mites. Similarly, the insecticide flubendiamide, which is also based on modification of carboxamides, was also discovered and developed as the first commercial phthalic diamide insecticide by Nihon Nohyaku Co. Ltd.; however, flubendiamide targets the insect ryanodine receptor rather than mitochondrial complex II in the respiratory chain [18,19]. Subsequently, chlorantraniliprole, another insecticide that acts on the insect ryanodine receptor was developed. Chlorantraniliprole contains an anthranilic diamide skeleton and was initially discovered through converting the amide bond connection of phthalic diamides [20]. Inspired by this strategy and by the excellent acaricidal and insecticidal activities of diamide compounds, we were motivated to explore new bioactive compounds by modifying the structure of pyflubumide. 
In most SDHI fungicides, two aromatic moieties are connected directly via a carboxamide. However, isofetamid C (Figure 1) and fluopyram D (Figure 1), in which two aromatic moieties are bridged by an $\mathrm{N}$-ethyl carboxamide or $\mathrm{N}$-oxo-ethyl carboxamide, also exhibit excellent fungicidal activity, demonstrating the possibility of discovering new compounds by modifying the connection between the two aromatic moieties [21,22].

Inspired by the design of chlorantraniliprole from flubendiamide, pyflubumide was selected as the lead compound, and a series of novel 1,3,5-trimethylpyrazole-containing malonamide derivatives were designed and synthesized (Figure 2). The malonamide skeletons of the new compounds were designed by modifying two amide bonds on one nitrogen atom, and the phenyl ring was modified with a heptafluoroisopropyl group or a methoxy-substituted hexafluoroisopropyl group to improve activity based on previous reports [23]. Moreover, all the new compounds were investigated for their acaricidal and insecticidal activities. To the best of our knowledge, it is the first report on 1,3,5-trimethylpyrazole-containing malonamide derivatives with potent acaricidal and insecticidal activities.

\section{Results and Discussion}

\subsection{Synthesis}

The synthetic route to the target compounds $\mathbf{8 a} \sim \mathbf{8 t}$ is outlined in Scheme 1; these compounds were prepared in four steps. The commercially available ethyl 3-chloro-3-oxopropanoate (1) was reacted with the aromatic amine derivative 2 in the presence of triethylamine to afford the intermediate 3 via a general condensation reaction in good yields. Intermediate 3 was hydrolyzed to obtain intermediate 4 , which was used in the next reaction without further purification. In the presence of the condensation agent dicyclohexylcarbodiimide (DCC), intermediate 4 was reacted with 1,3,5-trimethyl-1H-pyrazol-4-amine (5) to afford the key intermediate 6 in good yields. The last step was $\alpha$-alkylation of malonamide with brominated compound 7 in the presence of potassium t-butoxide (KTB) to obtain the target compounds 8a-8t [24]. Then the reaction condition of the last step was investigated to optimize the yield. The examination of the base, solvent, and temperature led to the following informative observations: (i) potassium t-butoxide was the ideal base; (ii) $N$-methyl pyrrolidone (NMP) was the suitable solvent; (iii) the product yield was highest at 0 to $5{ }^{\circ} \mathrm{C}$. It was observed that the steric effect and electronic effect of substituents $\left(\mathrm{R}_{2}\right)$ had a significant effect on the yield of target compounds. For example, with the isopentyl group, compound $8 \mathrm{c}$ was $48.4 \%$ yield. Otherwise, compound 8e with allyl group was $61.7 \%$ yield. The target compounds have all been structurally confirmed through ${ }^{1} \mathrm{H}-\mathrm{NMR},{ }^{13} \mathrm{C}-\mathrm{NMR}$ and high-resolution mass spectra (HRMS). 


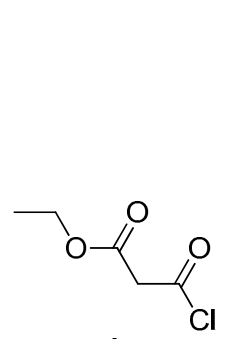

1

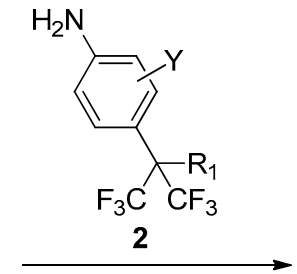

(a)

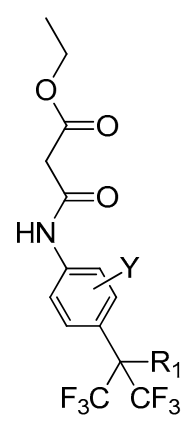

3

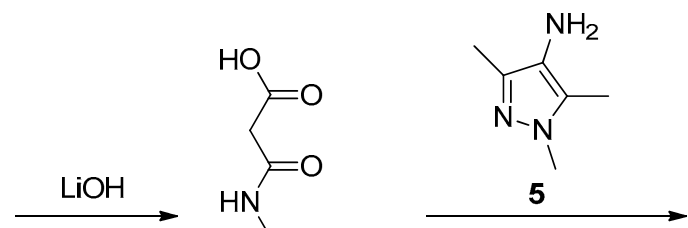

(b)

(c)<smiles>[Y]c1ccc(C([R7])(C(F)(F)F)C(F)(F)F)cc1NC(=O)CC(=O)Nc1c(C)nn(C)c1C</smiles>

6

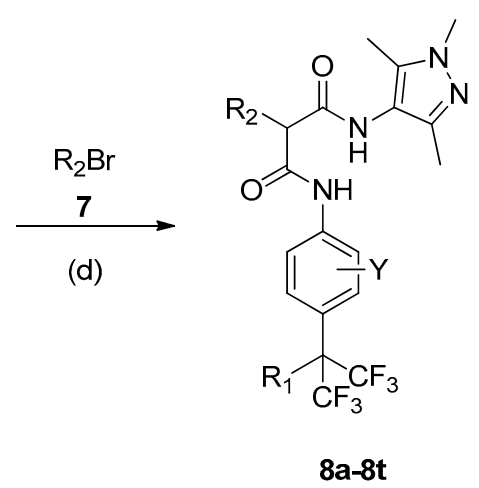

8b: $\mathrm{R}_{1}=\mathrm{F}, \mathrm{R}_{2}=$ Isobutyl, $\mathrm{Y}=2-\mathrm{Me}$

8d: $R_{1}=F, R_{2}=$ Propargyl, $Y=2-M e$

8f: $\mathrm{R}_{1}=\mathrm{OMe}, \mathrm{R}_{2}=$ Isopropyl, $\mathrm{Y}=2-\mathrm{Me}$

8h: $\mathrm{R}_{1}=\mathrm{OMe}, \mathrm{R}_{2}=$ Isopentyl, $\mathrm{Y}=2-\mathrm{Me}$

8j: $\mathrm{R}_{1}=\mathrm{OMe}, \mathrm{R}_{2}=$ Allyl, $\mathrm{Y}=2-\mathrm{Me}$

8I: $\mathrm{R}_{1}=\mathrm{OMe}, \mathrm{R}_{2}=$ Isobutyl, $\mathrm{Y}=2$-OMe

8n: $\mathrm{R}_{1}=\mathrm{OMe}, \mathrm{R}_{2}=$ Propargyl, $\mathrm{Y}=2-\mathrm{OMe}$

8p: $\mathrm{R}_{1}=\mathrm{OMe}, \mathrm{R}_{2}=$ Isopropyl, $\mathrm{Y}=3$-Isopropyl

8r: $\mathrm{R}_{1}=\mathrm{OMe}, \mathrm{R}_{2}=$ Isopentyl, $\mathrm{Y}=$ 3-Isopropyl

8t: $\mathrm{R}_{1}=\mathrm{OMe}, \mathrm{R}_{2}=$ Propargyl, $\mathrm{Y}=3$-Isopropyl

Scheme 1. General synthetic route for compounds 8a-8t. Reagents and conditions: (a) $\mathrm{Et}_{3} \mathrm{~N}, \mathrm{CH}_{2} \mathrm{Cl}_{2}$, 0-5 ${ }^{\circ} \mathrm{C}$; (b) $\mathrm{LiOH}, \mathrm{H}_{2} \mathrm{O}$, THF, 0-25 ${ }^{\circ} \mathrm{C}$; (c) DCC, $\mathrm{CH}_{2} \mathrm{Cl}_{2}, 0-25{ }^{\circ} \mathrm{C}$; (d) KTB, NMP, 0-25 ${ }^{\circ} \mathrm{C}$.

\subsection{Biological Activity}

As the primary objective of this study, preliminary tests of the acaricidal and insecticidal activity of the target compounds 8a-8t against the pest species Tetranychus cinnabarinus, Plutella xylostella, and Aphis craccivora were carried out, as shown in Tables 1-3.

First, the acaricidal activity of the target compounds $\mathbf{8 a - 8 t}$ against Tetranychus cinnabarinus was carried out, and the results were summarized in Table 1 . Fenpyroximate was tested under the same conditions as a comparison compound. As indicated in Table 1, all new compounds exhibited certain acaricidal activity against Tetranychus cinnabarinus, with $\mathrm{LC}_{50}$ values ranging from 107.2 to $540.4 \mu \mathrm{g} / \mathrm{mL}$. Among the target compounds, $\mathbf{8 a}, \mathbf{8 i - 8 k}, \mathbf{8 m}-\mathbf{8 n}$, and $\mathbf{8 p}-\mathbf{8 r}$ exhibited good acaricidal activities against Tetranychus cinnabarinus with more than $90.0 \%$ mortality at $400 \mu \mathrm{g} / \mathrm{mL}$, respectively, which were comparable to that of the control fenpyroximate $(100.0 \%)$. When the concentrations of the compounds were reduced from 400 to $200 \mu \mathrm{g} / \mathrm{mL}$, compounds $8 \mathrm{~m}$ and $8 \mathrm{p}$ still showed good mortality $(70.0 \%)$ against Tetranychus cinnabarinus. However, a great decrease of bioactivities (0-37.8\%) was observed, when the concentrations were reduced from 200 to $100 \mu \mathrm{g} / \mathrm{mL}$. The activities of these compounds 
varied drastically depending upon the types and patterns of substitution on the malonamide bridge and the phenyl ring.

For the effect of substituents $R_{2}$ on the malonamide bridge in the series of $\mathbf{8} \mathbf{a} \sim \mathbf{8 t}$ compounds, when the substituent $\mathrm{R}_{2}$ was saturated alkyl (isopropyl, isobutyl, and isopentyl), it was observed that compounds with isopropyl group, like $\mathbf{8 a}, \mathbf{8 f}, \mathbf{8 k}$, and $\mathbf{8 p}$, exhibited higher acaricidal potency than the corresponding analog with isobutyl groups, such as $\mathbf{8 b}, \mathbf{8 g}, \mathbf{8 1}$, and $\mathbf{8 q}$. Meanwhile, compared with compounds with isobutyl group, compounds with isopentyl (8h, 8m, 8r, except 8c) revealed more activities. When the substituent $\mathrm{R}_{2}$ was unsaturated alkyl (propargyl and allyl), it was observed that compounds with propargyl group, like $\mathbf{8 d}, \mathbf{8 i}, \mathbf{8 n}$, and $\mathbf{8 s}$, exhibited higher acaricidal potency than the corresponding analog with allyl groups, such as $\mathbf{8 e}, \mathbf{8 j}, \mathbf{8 0}$, and $\mathbf{8 t}$. On the whole, the introduction of saturated alkyl with appropriate chain length at position $R_{2}$ was beneficial to the acaricidal activity.

For the effect of substituents on the phenyl ring in the series of $\mathbf{8 a - 8 t}$ compounds, from the activities against Tetranychus cinnabarinus, the compounds $\mathbf{8 f}-\mathbf{8 t}$ with the methoxy-substituted hexafluoroisopropyl group gave increased acaricidal activity than the compounds $\mathbf{8 a}-\mathbf{8 e}$ with the heptafluoroisopropyl group, respectively. In addition, when $\mathrm{R}_{2}$ was saturated alkyl, the compounds (8k-8m) with methoxy group on the 2-position of phenyl ring displayed relatively better acaricidal activity than the corresponding compounds $(\mathbf{8 f}-\mathbf{8 h})$ with a methyl group on the 2-position of the phenyl ring.

Table 1. Insecticidal activities of compounds 8a $\sim \mathbf{8 t}$ and fenpyroximate (FEN) against Tetranychus cinnabarinus.

\begin{tabular}{|c|c|c|c|c|c|c|c|}
\hline Compound & \multicolumn{5}{|c|}{ Mortality (\%) ${ }^{a}$} & $\begin{array}{c}\mathrm{LC}_{50} \\
(\mu \mathrm{g} / \mathrm{mL})\end{array}$ & $\begin{array}{l}95 \% \mathrm{CL} \\
(\mu \mathrm{g} / \mathrm{mL})\end{array}$ \\
\hline $8 a$ & $90.0 \pm 3.3^{b}$ & $60.0 \pm 5.8$ & $24.4 \pm 1.9$ & $11.1 \pm 1.9$ & $0.0 \pm 0.0$ & 157.2 & 127.5-197.0 \\
\hline $8 c$ & $60.0 \pm 3.3$ & $35.6 \pm 1.9$ & $0.0 \pm 0.0$ & $0.0 \pm 0.0$ & $0.0 \pm 0.0$ & 308.8 & $254.2-405.3$ \\
\hline $8 d$ & $61.1 \pm 3.8$ & $21.1 \pm 1.9$ & $0.0 \pm 0.0$ & $0.0 \pm 0.0$ & $0.0 \pm 0.0$ & 333.1 & $278.5-431.0$ \\
\hline $8 g$ & $51.1 \pm 3.8$ & $20.0 \pm 3.3$ & $0.0 \pm 0.0$ & $0.0 \pm 0.0$ & $0.0 \pm 0.0$ & 376.7 & $305.6-544.9$ \\
\hline $8 \mathrm{~h}$ & $78.9 \pm 3.8$ & $41.1 \pm 1.9$ & $0.0 \pm 0.0$ & $0.0 \pm 0.0$ & $0.0 \pm 0.0$ & 250.7 & 212.9-298.7 \\
\hline $8 \mathrm{i}$ & $100.0 \pm 0.0$ & $64.4 \pm 1.9$ & $30.0 \pm 3.3$ & $17.8 \pm 1.9$ & $7.8 \pm 1.9$ & 119.2 & $52.0-343.6$ \\
\hline $8 \mathbf{j}$ & $90.0 \pm 3.3$ & $54.4 \pm 1.9$ & $20.0 \pm 3.3$ & $7.8 \pm 1.9$ & $0.0 \pm 0.0$ & 172.2 & $140.8-214.8$ \\
\hline $8 \mathrm{k}$ & $100.0 \pm 0.0$ & $60.0 \pm 3.3$ & $20.0 \pm 0.0$ & $8.9 \pm 1.9$ & $0.0 \pm 0.0$ & 149.5 & 124.8-180.9 \\
\hline $8 p$ & $100.0 \pm 0.0$ & $70.0 \pm 3.3$ & $37.8 \pm 1.9$ & $15.6 \pm 1.9$ & $5.6 \pm 1.9$ & 112.2 & $90.2-140.4$ \\
\hline $8 q$ & $100.0 \pm 0.0$ & $50.0 \pm 3.3$ & $20.0 \pm 0.0$ & $5.6 \pm 1.9$ & $0.0 \pm 0.0$ & 164.0 & $103.3-288.0$ \\
\hline $8 r$ & $100.0 \pm 0.0$ & $60.0 \pm 0.0$ & $25.6 \pm 1.9$ & $12.2 \pm 1.9$ & $7.8 \pm 1.9$ & 132.3 & 39.7-1172.9 \\
\hline $8 s$ & $85.6 \pm 1.9$ & $43.3 \pm 3.3$ & $20.0 \pm 3.3$ & $8.9 \pm 1.9$ & $0.0 \pm 0.0$ & 194.2 & $156.0-252.1$ \\
\hline $8 t$ & $38.9 \pm 1.9$ & $18.9 \pm 3.8$ & $0.0 \pm 0.0$ & $0.0 \pm 0.0$ & $0.0 \pm 0.0$ & 460.3 & $348.5-879.2$ \\
\hline FEN & $100.0 \pm 0.0$ & $100.0 \pm 0.0$ & $100.0 \pm 0.0$ & $100.0 \pm 0.0$ & $100.0 \pm 0.0$ & - & - \\
\hline
\end{tabular}

${ }^{\mathrm{a}}$ Experimental size: 30 insects per group, three groups. ${ }^{\mathrm{b}}$ Each value represents the mean \pm standard deviation of three replications; $\mathrm{LC}_{50}=50 \%$ lethal concentration; $95 \% \mathrm{CL}=95 \%$ confidence limit; "- " refers to "not calculated".

Next, we evaluated their insecticidal activities against Plutella xylostella and Aphis craccivora, and the results are shown in Tables 2 and 3. Flubendiamide and imidacloprid were tested under the same conditions as a comparison compound.

As shown in Table 2, the bioactivity results indicated that most compounds exhibited certain insecticidal activities against Plutella xylostella, with $\mathrm{LC}_{50}$ values ranging from 24.2 to $569.8 \mu \mathrm{g} / \mathrm{mL}$. Among the target compounds, some compounds possessed excellent insecticidal activities against Plutella xylostella at a concentration of $200 \mu \mathrm{g} / \mathrm{mL}$. For instance, the mortalities of compounds $\mathbf{8 g}$, 8i, and 80 against Plutella xylostella were all 100.0\%, respectively, which were similar to that of the 
control flubendiamide. When the concentration was reduced to $100 \mu \mathrm{g} / \mathrm{mL}$, compounds $\mathbf{8 i}$ and $8 \mathbf{0}$ had $100.0 \%$ inhibition rates, and they were still active against Plutella xylostella even when the concentration was reduced to $50 \mu \mathrm{g} / \mathrm{mL}$ with inhibitory values of $83.3 \%$ and $76.7 \%$, respectively. Based on the structure-activity data, we found that unsaturated alkyl (propargyl and allyl) on the position $R_{2}$ were beneficial to improve the insecticidal activity of compounds, for example, compounds $\mathbf{8 e}, \mathbf{8 i}, \mathbf{8 0}$, and $\mathbf{8 s}$ were the most active of the corresponding series of compounds $\mathbf{8 a}-\mathbf{8 e}, \mathbf{8 f}-\mathbf{8 j}, \mathbf{8 k}-\mathbf{8 o}$, and $\mathbf{8 p}-\mathbf{8 t}$, respectively. In addition, we found that when the substituent at 4-position of the phenyl ring was a methoxy-substituted hexafluoroisopropyl group, it was more advantageous than heptafluoroisopropyl group to increase the insecticidal activities against Plutella xylostella. Moreover, compared with compounds (8p-8t) with the substituent (isopropyl group) at 3-position of the phenyl ring, compounds (8a-8o) with the substituent (methyl and methoxy groups) at 2-position of phenyl ring showed more advantages in insecticidal activity.

Table 2. Insecticidal activities of compounds 8a-8t and flubendiamide (FLU) against Plutella xylostellas.

\begin{tabular}{|c|c|c|c|c|c|c|c|}
\hline Compound & \multicolumn{5}{|c|}{ Mortality (\%) } & $\begin{array}{c}\mathrm{LC}_{50} \\
(\mu \mathrm{g} / \mathrm{mL})\end{array}$ & $\begin{array}{l}95 \% \mathrm{CL} \\
(\mu \mathrm{g} / \mathrm{mL})\end{array}$ \\
\hline $8 a$ & $80.0 \pm 10.0^{b}$ & $60.0 \pm 0.0$ & $30.0 \pm 10.0$ & $16.7 \pm 5.8$ & $10.0 \pm 0.0$ & 153.2 & 89.8-333.2 \\
\hline $8 b$ & $53.3 \pm 5.8$ & $30.0 \pm 0.0$ & $16.7 \pm 5.8$ & $6.7 \pm 5.8$ & $0.0 \pm 0.0$ & 359.0 & $204.7-2371.2$ \\
\hline $8 c$ & $63.3 \pm 5.8$ & $36.7 \pm 5.8$ & $10.0 \pm 0.0$ & $6.7 \pm 5.8$ & $0.0 \pm 0.0$ & 289.3 & $183.8-808.9$ \\
\hline $8 d$ & $90.0 \pm 0.0$ & $73.3 \pm 5.8$ & $30.0 \pm 0.0$ & $23.3 \pm 5.8$ & $16.7 \pm 5.8$ & 109.5 & $63.0-204.2$ \\
\hline $8 g$ & $100.0 \pm 0.0$ & $100.0 \pm 0.0$ & $43.3 \pm 5.8$ & $20.0 \pm 0.0$ & $6.7 \pm 5.8$ & 82.6 & 57.2-119.3 \\
\hline $8 \mathrm{~h}$ & $86.7 \pm 5.8$ & $60.0 \pm 0.0$ & $30.0 \pm 10.0$ & $13.3 \pm 5.8$ & $0.0 \pm 0.0$ & 154.0 & $102.2-250.5$ \\
\hline $8 i$ & $100.0 \pm 0.0$ & $100.0 \pm 0.0$ & $100.0 \pm 0.0$ & $83.3 \pm 5.8$ & $46.7 \pm 5.8$ & 22.1 & $6.6-33.3$ \\
\hline $8 \mathrm{j}$ & $70.0 \pm 0.0$ & $33.3 \pm 5.8$ & $20.0 \pm 0.0$ & $6.7 \pm 5.8$ & $0.0 \pm 0.0$ & 257.3 & $164.4-636.1$ \\
\hline $8 k$ & $60.0 \pm 0.0$ & $50.0 \pm 0.0$ & $30.0 \pm 0.0$ & $16.7 \pm 5.8$ & $6.7 \pm 5.8$ & 235.4 & $124.0-1322.9$ \\
\hline 80 & $100.0 \pm 0.0$ & $100.0 \pm 0.0$ & $100.0 \pm 0.0$ & $76.7 \pm 5.8$ & $43.3 \pm 5.8$ & 24.2 & 8.9-36.5 \\
\hline $8 p$ & $30.0 \pm 0.0$ & $10.0 \pm 0.0$ & $0.0 \pm 0.0$ & $0.0 \pm 0.0$ & $0.0 \pm 0.0$ & 569.8 & $405.6-2013.3$ \\
\hline $8 q$ & $76.7 \pm 5.8$ & $40.0 \pm 0.0$ & $20.0 \pm 0.0$ & $10.0 \pm 0.0$ & $0.0 \pm 0.0$ & 220.0 & $142.9-454.3$ \\
\hline $8 r$ & $56.7 \pm 5.8$ & $26.7 \pm 5.8$ & $10.0 \pm 0.0$ & $6.7 \pm 5.8$ & $0.0 \pm 0.0$ & 359.0 & 213.2-1857.2 \\
\hline $8 s$ & $90.0 \pm 0.0$ & $56.7 \pm 5.8$ & $26.7 \pm 5.8$ & $13.3 \pm 5.8$ & $6.7 \pm 5.8$ & 149.9 & $95.6-268.6$ \\
\hline $8 \mathbf{t}$ & $30.0 \pm 0.0$ & $0.0 \pm 0.0$ & $0.0 \pm 0.0$ & $0.0 \pm 0.0$ & $0.0 \pm 0.0$ & - & - \\
\hline FLU & $100.0 \pm 0.0$ & $100.0 \pm 0.0$ & $100.0 \pm 0.0$ & $100.0 \pm 0.0$ & $100.0 \pm 0.0$ & - & - \\
\hline
\end{tabular}

${ }^{a}$ Experimental size: 10 insects per group, three groups. ${ }^{b}$ Each value represents the mean \pm standard deviation of three replications; $\mathrm{LC}_{50}=50 \%$ lethal concentration; $95 \% \mathrm{CL}=95 \%$ confidence limit; "- " refers to "not calculated".

As shown in Table 3, the bioactivity results indicated that all compounds exhibited certain insecticidal activities against Aphis craccivora, with $\mathrm{LC}_{50}$ values ranging from 8.1 to $225.3 \mu \mathrm{g} / \mathrm{mL}$. As can be seen, some target compounds demonstrated excellent insecticidal activities against Aphis craccivora at a concentration of $100 \mu \mathrm{g} / \mathrm{mL}$, for instance, compounds $\mathbf{8 a}, \mathbf{8 b}, \mathbf{8 c}, \mathbf{8 j}, \mathbf{8 k}, \mathbf{8 p}$, and $\mathbf{8 q}$ all had over $90.0 \%$ inhibition rates, which were comparable to that of the control imidacloprid (100.0\%). When the concentration was reduced to $50 \mu \mathrm{g} / \mathrm{mL}$, the mortalities of compounds $\mathbf{8 a}, \mathbf{8 k}, \mathbf{8 p}$, and $\mathbf{8 q}$ were $80.0 \%, 76.7 \%, 100.0 \%$, and $100.0 \%$, respectively. Even if the concentration was reduced to $20 \mu \mathrm{g} / \mathrm{mL}$, compounds $\mathbf{8 p}$ and $\mathbf{8 q}$ were still active against Aphis craccivora with the mortalities of $88.3 \%$ and $83.3 \%$, respectively. Based on the structure-activity data, we found that the saturated alkyl on the position $\mathrm{R}_{2}$ was more helpful to increase the mortality activities against Aphis craccivora than the unsaturated alkyl. Especially, when $\mathrm{R}_{2}$ substituent group was an isopropyl group, it showed better insecticidal activities against Aphis craccivora than other types of $\mathrm{R}_{2}$ substituents. In addition, compared with compounds (8a-8e) with the substituent heptafluoroisopropyl group at 4-position of the phenyl ring, compounds (8f-8t) with the substituent methoxy-substituted hexafluoroisopropyl group showed more advantages in insecticidal activity. Moreover, we found that the introduction of isopropyl group 
at 3-position of the phenyl ring influenced the insecticidal activities greatly, for example, compounds $\mathbf{8 p}$ and $\mathbf{8 q}$ were a more effective insecticidal agent than other compounds.

Table 3. Insecticidal activities of compounds 8a $\mathbf{8 t}$ and imidacloprid (IMI) against Aphis craccivora.

\begin{tabular}{|c|c|c|c|c|c|c|c|}
\hline \multirow{2}{*}{ Compound } & \multicolumn{5}{|c|}{ Mortality (\%) ${ }^{a}$} & \multirow{2}{*}{$\begin{array}{c}\mathrm{LC}_{50} \\
(\mu \mathrm{g} / \mathrm{mL})\end{array}$} & \multirow{2}{*}{$\begin{array}{l}95 \% \mathrm{CL} \\
(\mu \mathrm{g} / \mathrm{mL})\end{array}$} \\
\hline & $200 \mu \mathrm{g} / \mathrm{mL}$ & $100 \mu \mathrm{g} / \mathrm{mL}$ & $50 \mu \mathrm{g} / \mathrm{mL}$ & $20 \mu \mathrm{g} / \mathrm{mL}$ & $10 \mu \mathrm{g} / \mathrm{mL}$ & & \\
\hline $8 a$ & $100.0 \pm 0.0^{b}$ & $100.0 \pm 0.0$ & $80.0 \pm 0.0$ & $56.7 \pm 2.9$ & $21.7 \pm 2.9$ & 19.1 & $13.5-25.4$ \\
\hline $8 b$ & $100.0 \pm 0.0$ & $100.0 \pm 0.0$ & $70.0 \pm 0.0$ & $43.3 \pm 2.9$ & $20.0 \pm 5.0$ & 23.3 & $16.8-31.1$ \\
\hline $8 c$ & $100.0 \pm 0.0$ & $90.0 \pm 0.0$ & $65.0 \pm 0.0$ & $36.7 \pm 2.9$ & $15.0 \pm 0.0$ & 29.0 & $20.8-39.1$ \\
\hline $8 d$ & $71.7 \pm 2.9$ & $45.0 \pm 0.0$ & $20.0 \pm 0.0$ & $13.3 \pm 2.9$ & $8.3 \pm 2.9$ & 111.7 & $73.0-224.0$ \\
\hline $8 e$ & $40.0 \pm 0.0$ & $20.0 \pm 0.0$ & $0.0 \pm 0.0$ & $0.0 \pm 0.0$ & $0.0 \pm 0.0$ & 225.3 & $162.9-612.0$ \\
\hline $8 f$ & $100.0 \pm 0.0$ & $81.7 \pm 2.9$ & $58.3 \pm 2.9$ & $31.7 \pm 2.9$ & $18.3 \pm 2.9$ & 32.6 & $22.7-45.1$ \\
\hline $8 \mathrm{~g}$ & $90.0 \pm 0.0$ & $70.0 \pm 0.0$ & $50.0 \pm 5.0$ & $26.7 \pm 2.9$ & $11.7 \pm 2.9$ & 46.6 & $32.0-68.0$ \\
\hline $8 \mathrm{~h}$ & $80.0 \pm 5.0$ & $50.0 \pm 5.0$ & $30.0 \pm 0.0$ & $13.3 \pm 2.9$ & $8.3 \pm 2.9$ & 85.0 & 58.3-141.1 \\
\hline $8 \mathbf{i}$ & $100.0 \pm 0.0$ & $75.0 \pm 5.0$ & $50.0 \pm 0.0$ & $28.3 \pm 5.8$ & $16.7 \pm 2.9$ & 38.3 & $26.8-53.7$ \\
\hline $8 \mathbf{j}$ & $100.0 \pm 0.0$ & $91.7 \pm 2.9$ & $60.0 \pm 5.0$ & $36.7 \pm 2.9$ & $21.7 \pm 2.9$ & 27.6 & $19.2-37.9$ \\
\hline $8 k$ & $100.0 \pm 0.0$ & $100.0 \pm 0.0$ & $76.7 \pm 2.9$ & $41.7 \pm 2.9$ & $25.0 \pm 0.0$ & 21.4 & $15.2-28.5$ \\
\hline 81 & $71.7 \pm 2.9$ & $50.0 \pm 5.0$ & $40.0 \pm 0.0$ & $26.7 \pm 2.9$ & $13.3 \pm 2.9$ & 79.2 & $47.6-173.5$ \\
\hline $8 m$ & $100.0 \pm 0.0$ & $80.0 \pm 0.0$ & $50.0 \pm 5.0$ & $30.0 \pm 5.0$ & $18.3 \pm 2.9$ & 35.7 & $24.9-49.8$ \\
\hline $8 n$ & $90.0 \pm 0.0$ & $68.3 \pm 2.9$ & $50.0 \pm 5.0$ & $30.0 \pm 0.0$ & $18.3 \pm 2.9$ & 42.9 & $27.8-65.3$ \\
\hline 80 & $75.0 \pm 0.0$ & $50.0 \pm 5.0$ & $30.0 \pm 0.0$ & $18.3 \pm 2.9$ & $10.0 \pm 0.0$ & 88.4 & $57.7-166.1$ \\
\hline $8 p$ & $100.0 \pm 0.0$ & $100.0 \pm 0.0$ & $100.0 \pm 0.0$ & $88.3 \pm 2.9$ & $63.3 \pm 2.9$ & 8.1 & $1.8-11.3$ \\
\hline $8 q$ & $100.0 \pm 0.0$ & $100.0 \pm 0.0$ & $100.0 \pm 0.0$ & $83.3 \pm 2.9$ & $63.3 \pm 2.9$ & 8.1 & $2.1-11.6$ \\
\hline $8 r$ & $100.0 \pm 0.0$ & $70.0 \pm 5.0$ & $60.0 \pm 0.0$ & $43.3 \pm 2.9$ & $20.0 \pm 5.0$ & 30.9 & $19.7-44.9$ \\
\hline $8 \mathrm{~s}$ & $90.0 \pm 5.0$ & $65.0 \pm 5.0$ & $45.0 \pm 0.0$ & $25.0 \pm 0.0$ & $13.3 \pm 2.9$ & 50.7 & $34.5-76.0$ \\
\hline $8 \mathrm{t}$ & $50.0 \pm 5.0$ & $30.0 \pm 5.0$ & $0.0 \pm 0.0$ & $0.0 \pm 0.0$ & $0.0 \pm 0.0$ & 181.2 & $137.8-320.3$ \\
\hline IMI & $100.0 \pm 0.0$ & $100.0 \pm 0.0$ & $100.0 \pm 0.0$ & $100.0 \pm 0.0$ & $100.0 \pm 0.0$ & - & - \\
\hline
\end{tabular}

${ }^{\mathrm{a}}$ Experimental size: 20 insects per group, three groups. ${ }^{\mathrm{b}}$ Each value represents the mean \pm standard deviation of three replications; $\mathrm{LC}_{50}=50 \%$ lethal concentration; $95 \% \mathrm{CL}=95 \%$ confidence limit; "-" refers to "not calculated".

\section{Experimental}

\subsection{Chemicals and Instrumentation}

Nuclear magnetic resonance spectra were recorded using a Bruker Avance DPX300 (San Jose, CA, USA) spectrometer in $\mathrm{CDCl}_{3}$ or $\mathrm{DMSO}-\mathrm{d}_{6}$ solution $\left({ }^{1} \mathrm{H}-\mathrm{NMR}\right.$ at $300 \mathrm{MHz}$ and ${ }^{13} \mathrm{C}-\mathrm{NMR}$ at $75 \mathrm{MHz}$ ). Chemical shift values $(\delta)$ are given in parts per million with tetramethylsilane as the internal standard. The melting points of all compounds were determined using a B-III microscope (Beijing Technical Instrument Co., Beijing, China) and are uncorrected. High-resolution mass spectra (HRMS) were obtained using a Thermo Scientific Q Exactive instrument. Analytical TLC was performed on silica gel $\mathrm{GF}_{254}$ (200-300 mesh). Column chromatographic purification was carried out using silica gel.

Reagents and solvents were obtained from Beijing Chemical Reagents Co. (Beijing, China) and were used without purification. As shown in Scheme 1, ethyl malonyl chloride (compound 1), 1,3,5-trimethyl-1H-pyrazol-4-amine (compound 5), and compound 7 were purchased from Shanghai Aladdin Bio-Chem Technology Co., LTD. Compound 2 was prepared according to literature methods without purification $[25,26]$.

\subsection{Synthesis Procedures}

\subsubsection{General synthesis Procedure for Intermediate 3}

A solution of intermediate $2(60 \mathrm{mmol})$ and triethylamine $(60 \mathrm{mmol})$ dissolved in dichloromethane (100 mL) was cooled to $0-5{ }^{\circ} \mathrm{C}$. Ethyl malonyl chloride $(60 \mathrm{mmol})$ was added dropwise over ten minutes, and the mixture was stirred for a further thirty minutes at this temperature. The reaction mixture was then stirred at room temperature until the reaction was complete as indicated by TLC. At the end of the reaction, the reaction mixture was successively washed with diluted hydrochloric acid (30 $\mathrm{mL}$, $5 \%$ vol) and brine, dried over anhydrous sodium sulfate, and concentrated in vacuo. The residue was 
purified by flash chromatography on silica gel using petroleum ether $\left(60-90{ }^{\circ} \mathrm{C}\right)$ and ethyl acetate $(v / v=15: 1)$ as the eluent to give intermediate 3 , with yields ranging from $89.4 \%$ to $93.5 \%$. Physical, spectral data, and ${ }^{1} \mathrm{H}-\mathrm{NMR}$ spectra of intermediate 3 were provided in the Supplementary Materials.

\subsubsection{General Synthesis Procedure for Intermediate 4}

To a solution of intermediate $3(20 \mathrm{mmol})$ in tetrahydrofuran $(50 \mathrm{~mL})$ was added $\mathrm{LiOH}$ $(2 \mathrm{~mol} / \mathrm{L}, 60 \mathrm{~mL})$ dropwise at $0^{\circ} \mathrm{C}$, and the reaction mixture was then warmed to room temperature and stirred overnight until the reaction was completed as indicated by TLC. The solvent was vacuum-evaporated, the residue was acidified with diluted hydrochloric acid ( $2 \mathrm{M}, 20 \mathrm{~mL})$, and the aqueous layer was extracted with dichloromethane $(30 \mathrm{~mL} \times 3)$. The combined organic phase was dried over anhydrous sodium sulfate and then concentrated in vacuo to give intermediate 4 , which was used in the next reaction without further purification.

\subsubsection{General Synthesis Procedure for Intermediate 6}

To a mixture of intermediate 4 (15 mmol) and 1,3,5-trimethyl- $1 H$-pyrazol-4-amine (15 mmol) in dichloromethane $(50 \mathrm{~mL})$ was added dicyclohexylcarbodiimide (DCC, $22.5 \mathrm{mmol})$ in dichloromethane $(20 \mathrm{~mL})$ dropwise at $0{ }^{\circ} \mathrm{C}$. The reaction mixture was then warmed to room temperature and stirred overnight until the reaction was completed. The mixture was then filtered, and the filtrate was washed successively with saturated sodium bicarbonate solution and saturated brine, dried over anhydrous sodium sulfate, and concentrated in vacuo. The residue was purified by flash chromatography on silica gel using petroleum ether $\left(60-90^{\circ} \mathrm{C}\right)$ and ethyl acetate $(v / v=1: 1)$ as the eluent to give intermediate 6 , with yields ranging from $82.5 \%$ to $86.7 \%$. Physical, spectral data, and ${ }^{1} \mathrm{H}-\mathrm{NMR}$ spectra of intermediate 6 were provided in the Supplementary Materials.

\subsubsection{General Synthesis Procedure for the Target Compounds 8a-8t}

To a solution of intermediate 6 ( $4 \mathrm{mmol})$ in $N$-methyl pyrrolidone (NMP) was added potassium $\mathrm{t}$-butoxide $(\mathrm{KTB}, 4.4 \mathrm{mmol})$ in portions at $0{ }^{\circ} \mathrm{C}$. After $30 \mathrm{~min}$, intermediate $7(4.4 \mathrm{mmol})$ was added at $0{ }^{\circ} \mathrm{C}$. The mixture was stirred $6 \mathrm{~h}$ at room temperature and then poured into ice-water and extracted with ethyl acetate $(30 \mathrm{~mL} \times 3)$. The combined organic phase was washed with saturated brine $(30 \mathrm{~mL}$ $\times 3$ ), dried over anhydrous sodium sulfate, and concentrated in vacuo. The residue was purified by flash chromatography on silica gel using petroleum ether $\left(60-90^{\circ} \mathrm{C}\right)$ and ethyl acetate $(v / v=1: 3)$ as the eluent to give the target compounds 8a $\mathbf{8 t}$, with yields ranging from $46.7 \%$ to $62.5 \%$. All twenty 1,3,5-trimethylpyrazole-containing malonamide derivatives 8a $\sim \mathbf{8 t}$ were novel, and their physical and spectral data are listed below. ${ }^{1} \mathrm{H}-\mathrm{NMR},{ }^{13} \mathrm{C}-\mathrm{NMR}$, and HRMS spectra are provided in the Supplementary Materials.

2-Isopropyl-N $N^{1}$-(2-methyl-4-(perfluoropropan-2-yl)phenyl)-N ${ }^{3}-(1,3,5$-trimethyl-1H-pyrazol-4-yl)malonamide (8a): White solid; m.p. 197-199 ${ }^{\circ} \mathrm{C}$; yield 53.2\%; ${ }^{1} \mathrm{H}-\mathrm{NMR}(300 \mathrm{MHz}$, Chloroform-d): $\delta 9.54(\mathrm{~s}, 1 \mathrm{H}$, pyrazole-NH), 8.51 (s, 1H, Ar-NH), 8.14 (d, J = 9.3 Hz, 1H, Ar-H), 7.43-7.41 (m, 2H, Ar-H), 3.69 $\left(\mathrm{s}, 3 \mathrm{H}, \mathrm{N}-\mathrm{CH}_{3}\right), 3.19(\mathrm{~d}, \mathrm{~J}=9.7 \mathrm{~Hz}, 1 \mathrm{H}, \mathrm{O}=\mathrm{C}-\mathrm{CH}-\mathrm{C}=\mathrm{O}), 2.47-2.39\left(\mathrm{~m}, 1 \mathrm{H}, \mathrm{CH}\left(\mathrm{CH}_{3}\right)_{2}\right), 2.35(\mathrm{~s}, 3 \mathrm{H}$, $\left.\mathrm{Ar}_{-} \mathrm{CH}_{3}\right), 2.11\left(\mathrm{~d}, J=1.2 \mathrm{~Hz}, 6 \mathrm{H}\right.$, pyrazole- $\left.\mathrm{CH}_{3}\right), 1.13\left(\mathrm{dd}, J=9.5,6.7 \mathrm{~Hz}, 6 \mathrm{H}, \mathrm{CH}\left(\mathrm{CH}_{3}\right)_{2}\right) .{ }^{13} \mathrm{C}-\mathrm{NMR}$ (75 MHz, Chloroform-d): $\delta 170.69(\mathrm{C}=\mathrm{O}), 168.38(\mathrm{C}=\mathrm{O}), 142.68(\mathrm{C}=\mathrm{N}$, pyrazole-C 3$), 137.95(\mathrm{C}-\mathrm{N}-\mathrm{N}$, pyrazole- $\left.\mathrm{C}_{5}\right), 134.47\left(\mathrm{C}-\mathrm{N}, \mathrm{Ar}-\mathrm{C}_{1}\right), 128.67\left(\mathrm{~d}, J_{\mathrm{CF}}=2.3 \mathrm{~Hz}, \mathrm{Ar}-\mathrm{C}_{2}\right), 127.34\left(\mathrm{~d}, J_{\mathrm{CF}}=11.3 \mathrm{~Hz}, \mathrm{Ar}-\mathrm{C}_{3}\right)$, $123.81\left(\mathrm{~d}, J_{\mathrm{CF}}=10.5 \mathrm{~Hz}, \mathrm{Ar}-\mathrm{C}_{5}\right), 122.15\left(\mathrm{~d}, J_{\mathrm{CF}}=20.3 \mathrm{~Hz}, \mathrm{Ar}-\mathrm{C}_{4}\right), 121.25\left(\mathrm{~d}, J_{\mathrm{CF}}=1.5 \mathrm{~Hz}, \mathrm{Ar}-\mathrm{C}_{6}\right), 120.22$ $\left(\mathrm{qd},{ }^{1} J_{\mathrm{CF}}=284.3 \mathrm{~Hz},{ }^{2} J_{\mathrm{CF}}=27.8 \mathrm{~Hz}, \mathrm{CF}_{3}\right), 113.67(\mathrm{C}-\mathrm{N}$, pyrazole-C 4$), 90.98\left(\mathrm{dsept},{ }^{1} J_{\mathrm{CF}}=200.3 \mathrm{~Hz}\right.$, $\left.\left.{ }^{2} J_{\mathrm{CF}}=33.0 \mathrm{~Hz}, \underline{\mathrm{CF}}\left(\mathrm{CF}_{3}\right)_{2}\right), 62.71(\mathrm{O}=\mathrm{C}-\underline{\mathrm{C}}-\mathrm{C}=\mathrm{O}), 35.90(\mathrm{~N}-\mathrm{C}, \text { pyrazole-CH})_{3}\right), 32.72\left(\underline{\mathrm{CH}}\left(\mathrm{CH}_{3}\right)_{2}\right), 20.53$ $\left(\mathrm{CH}\left(\mathrm{CH}_{3}\right)_{2}\right), 20.26\left(\mathrm{CH}\left(\mathrm{CH}_{3}\right)_{2}\right), 17.69\left(\mathrm{Ar}-\mathrm{CH}_{3}\right), 11.00\left(\right.$ pyrazole- $\left.\mathrm{CH}_{3}\right), 9.15$ (pyrazole-CH$) . \mathrm{HRMS}$ calcd. for $\mathrm{C}_{22} \mathrm{H}_{25} \mathrm{~F}_{7} \mathrm{~N}_{4} \mathrm{O}_{2}\left([\mathrm{M}+\mathrm{H}]^{+}\right)$, 511.1939; found, 511.1931.

2-Isobutyl-N $N^{1}-\left(2-m e t h y l-4-\left(\right.\right.$ perfluoropropan-2-yl)phenyl)-N $N^{3}-(1,3,5-$ trimethyl-1H-pyrazol-4-yl)malonamide (8b): White solid; m.p. 204-206 ${ }^{\circ} \mathrm{C}$; yield 56.5\%; ${ }^{1} \mathrm{H}-\mathrm{NMR}(300 \mathrm{MHz}$, Chloroform-d): $\delta 9.48$ (s, $1 \mathrm{H}$, 
pyrazole-NH), $8.41(\mathrm{~s}, 1 \mathrm{H}, \mathrm{Ar}-\mathrm{NH}), 8.11(\mathrm{~d}, J=9.3 \mathrm{~Hz}, 1 \mathrm{H}, \mathrm{Ar}-\mathrm{H}), 7.43-7.41(\mathrm{~m}, 2 \mathrm{H}, \mathrm{Ar}-\mathrm{H}), 3.69$ $\left(\mathrm{s}, 3 \mathrm{H}, \mathrm{N}-\mathrm{CH}_{3}\right), 3.63(\mathrm{t}, J=7.6 \mathrm{~Hz}, 1 \mathrm{H}, \mathrm{O}=\mathrm{C}-\mathrm{CH}-\mathrm{C}=\mathrm{O}), 2.35\left(\mathrm{~s}, 3 \mathrm{H}, \mathrm{Ar}^{-\mathrm{CH}_{3}}\right), 2.10(\mathrm{~d}, J=1.9 \mathrm{~Hz}, 6 \mathrm{H}$, pyrazole- $\left.\mathrm{CH}_{3}\right), 2.01-1.87\left(\mathrm{~m}, 2 \mathrm{H}, \mathrm{O}=\mathrm{C}-\mathrm{CH}-\mathrm{CH}_{2}\right), 1.80-1.71\left(\mathrm{~m}, 1 \mathrm{H}, \mathrm{CH}\left(\mathrm{CH}_{3}\right)_{2}\right), 1.00(\mathrm{~d}, J=6.5 \mathrm{~Hz}$, 6H, $\left.\mathrm{CH}\left(\mathrm{CH}_{3}\right)_{2}\right) .{ }^{13} \mathrm{C}-\mathrm{NMR}(75 \mathrm{MHz}$, Chloroform-d $): \delta 171.19(\mathrm{C}=\mathrm{O}), 169.13(\mathrm{C}=\mathrm{O}), 142.64(\mathrm{C}=\mathrm{N}$, pyrazole- $\left.\mathrm{C}_{3}\right), 137.89\left(\mathrm{C}-\mathrm{N}-\mathrm{N}\right.$, pyrazole- $\left.\mathrm{C}_{5}\right), 134.42\left(\mathrm{C}-\mathrm{N}, \mathrm{Ar}-\mathrm{C}_{1}\right), 128.81\left(\mathrm{~d}, J_{\mathrm{CF}}=2.3 \mathrm{~Hz}, \mathrm{Ar}-\mathrm{C}_{2}\right), 127.34$ $\left(\mathrm{d}, J_{\mathrm{CF}}=11.3 \mathrm{~Hz}, \mathrm{Ar}-\mathrm{C}_{3}\right), 123.79\left(\mathrm{~d}, J_{\mathrm{CF}}=10.5 \mathrm{~Hz}, \mathrm{Ar}-\mathrm{C}_{5}\right), 122.28\left(\mathrm{~d}, J_{\mathrm{CF}}=20.3 \mathrm{~Hz}, \mathrm{Ar}-\mathrm{C}_{4}\right), 121.43$ $\left(\mathrm{d}, J_{\mathrm{CF}}=0.8 \mathrm{~Hz}, \mathrm{Ar}-\mathrm{C}_{6}\right), 120.22\left(\mathrm{qd},{ }^{1} J_{\mathrm{CF}}=284.3 \mathrm{~Hz},{ }^{2} J_{\mathrm{CF}}=27.8 \mathrm{~Hz}, \mathrm{CF}_{3}\right), 113.60\left(\mathrm{C}-\mathrm{N}\right.$, pyrazole- $\left.\mathrm{C}_{4}\right)$, $90.97\left(\mathrm{dsept},{ }^{1} J_{\mathrm{CF}}=200.3 \mathrm{~Hz},{ }^{2} J_{\mathrm{CF}}=33.0 \mathrm{~Hz}, \underline{\mathrm{CF}}\left(\mathrm{CF}_{3}\right)_{2}\right), 53.53(\mathrm{O}=\mathrm{C}-\mathrm{C}-\mathrm{C}=\mathrm{O}), 42.50\left(\mathrm{O}=\mathrm{C}-\mathrm{CH}-\mathrm{CH}_{2}\right)$, $35.89\left(\mathrm{~N}-\mathrm{C}\right.$, pyrazole- $\left.\mathrm{CH}_{3}\right), 26.17\left(\mathrm{CH}\left(\mathrm{CH}_{3}\right)_{2}\right), 22.11\left(\mathrm{CH}\left(\mathrm{CH}_{3}\right)_{2}\right), 21.69\left(\mathrm{CH}\left(\mathrm{CH}_{3}\right)_{2}\right), 17.66\left(\mathrm{Ar}-\mathrm{CH}_{3}\right)$, 10.88 (pyrazole- $\left.\mathrm{CH}_{3}\right), 9.09$ (pyrazole- $\left.\mathrm{CH}_{3}\right)$. HRMS calcd. for $\mathrm{C}_{23} \mathrm{H}_{27} \mathrm{~F}_{7} \mathrm{~N}_{4} \mathrm{O}_{2}\left([\mathrm{M}+\mathrm{H}]^{+}\right), 525.2095$; found, 525.2086 .

2-Isopentyl-N ${ }^{1}$-(2-methyl-4-(perfluoropropan-2-yl)phenyl)- $N^{3}$-(1,3,5-trimethyl-1H-pyrazol-4-yl)malonamide (8c): Light yellow solid; m.p. 200-202 ${ }^{\circ} \mathrm{C}$; yield 48.4\%; ${ }^{1} \mathrm{H}-\mathrm{NMR}(300 \mathrm{MHz}$, Chloroform- $d$ ): $\delta 9.57$ (s, 1H, pyrazole-NH), $8.20(\mathrm{~s}, 1 \mathrm{H}, \mathrm{Ar}-\mathrm{NH}), 8.16(\mathrm{~d}, J=9.3 \mathrm{~Hz}, 1 \mathrm{H}, \mathrm{Ar}-\mathrm{H}), 7.44-7.42(\mathrm{~m}, 2 \mathrm{H}, \mathrm{Ar}-\mathrm{H}), 3.70$ $\left(\mathrm{s}, 3 \mathrm{H}, \mathrm{N}-\mathrm{CH}_{3}\right), 3.43(\mathrm{t}, J=7.5 \mathrm{~Hz}, 1 \mathrm{H}, \mathrm{O}=\mathrm{C}-\mathrm{CH}-\mathrm{C}=\mathrm{O}), 2.36\left(\mathrm{~s}, 3 \mathrm{H}, \mathrm{Ar}-\mathrm{CH}_{3}\right), 2.12\left(\mathrm{~s}, 6 \mathrm{H}\right.$, pyrazole- $\left.\mathrm{CH}_{3}\right)$, 2.09-2.03 (m, 2H, O=C-CH-CH $)_{2}, 1.66-1.57\left(\mathrm{~m}, 1 \mathrm{H}, \mathrm{CH}\left(\mathrm{CH}_{3}\right)_{2}\right), 1.41-1.33\left(\mathrm{~m}, 2 \mathrm{H}, \mathrm{CH}_{2}-\mathrm{CH}\left(\mathrm{CH}_{3}\right)_{2}\right)$, $0.89\left(\mathrm{~d}, J=6.6 \mathrm{~Hz}, 6 \mathrm{H}, \mathrm{CH}\left(\mathrm{CH}_{3}\right)_{2}\right) .{ }^{13} \mathrm{C}-\mathrm{NMR}(75 \mathrm{MHz}$, Chloroform- $d): \delta 171.29(\mathrm{C}=\mathrm{O}), 168.90(\mathrm{C}=\mathrm{O})$, $142.66\left(\mathrm{C}=\mathrm{N}\right.$, pyrazole- $\left.\mathrm{C}_{3}\right), 137.93\left(\mathrm{C}-\mathrm{N}-\mathrm{N}\right.$, pyrazole- $\left.\mathrm{C}_{5}\right), 134.48\left(\mathrm{C}-\mathrm{N}, \mathrm{Ar}-\mathrm{C}_{1}\right), 128.70\left(\mathrm{~d}, J_{\mathrm{CF}}=2.3 \mathrm{~Hz}\right.$, $\left.\operatorname{Ar}-C_{2}\right), 127.33\left(\mathrm{~d}, J_{\mathrm{CF}}=10.5 \mathrm{~Hz}, \mathrm{Ar}-\mathrm{C}_{3}\right), 123.82\left(\mathrm{~d}, J_{\mathrm{CF}}=10.5 \mathrm{~Hz}, \mathrm{Ar}_{-} \mathrm{C}_{5}\right), 122.21\left(\mathrm{~d}, J_{\mathrm{CF}}=20.3 \mathrm{~Hz}\right.$, Ar- $\left.C_{4}\right), 121.34\left(\mathrm{~d}, J_{\mathrm{CF}}=1.5 \mathrm{~Hz}, \mathrm{Ar}-\mathrm{C}_{6}\right), 120.22\left(\mathrm{qd},{ }^{1} J_{\mathrm{CF}}=285 \mathrm{~Hz},{ }^{2} J_{\mathrm{CF}}=28.5 \mathrm{~Hz}, \mathrm{CF}_{3}\right), 113.55(\mathrm{C}-\mathrm{N}$, pyrazole-C $\left.{ }_{4}\right), 90.98\left(\mathrm{dsept}^{1}{ }^{1} \mathrm{CF}_{\mathrm{CF}}=200.3 \mathrm{~Hz},{ }^{2} J_{\mathrm{CF}}=33.0 \mathrm{~Hz}, \underline{\mathrm{CF}}\left(\mathrm{CF}_{3}\right)_{2}\right), 55.15(\mathrm{O}=\mathrm{C}-\mathrm{C}-\mathrm{C}=\mathrm{O}), 36.31(\mathrm{~N}-\mathrm{C}$, pyrazole- $\left.\mathrm{CH}_{3}\right), 35.91\left(\mathrm{CH}_{2}-\mathrm{CH}\left(\mathrm{CH}_{3}\right)_{2}\right), 31.64\left(\underline{\mathrm{CH}}\left(\mathrm{CH}_{3}\right)_{2}\right), 27.39\left(\mathrm{O}=\mathrm{C}-\mathrm{CH}-\underline{\mathrm{C}} \mathrm{H}_{2}\right), 21.97\left(\mathrm{CH}\left(\mathrm{CH}_{3}\right)_{2}\right)$, $21.89\left(\mathrm{CH}\left(\mathrm{CH}_{3}\right)_{2}\right), 17.72\left(\mathrm{Ar}-\mathrm{CH}_{3}\right), 10.93$ (pyrazole- $\left.\mathrm{CH}_{3}\right), 9.13$ (pyrazole- $\left.\mathrm{CH}_{3}\right)$. HRMS calcd. for $\mathrm{C}_{24} \mathrm{H}_{29} \mathrm{~F}_{7} \mathrm{~N}_{4} \mathrm{O}_{2}\left([\mathrm{M}+\mathrm{H}]^{+}\right)$, 539.2252; found, 539.2244.

$N^{1}$-(2-Methyl-4-(perfluoropropan-2-yl)phenyl)-2-(prop-2-yn-1-yl)- $N^{3}-(1,3,5-$ trimethyl-1H-pyrazol-4-yl) malonamide (8d): White solid; m.p. $83-85{ }^{\circ} \mathrm{C}$; yield 50.2\%; ${ }^{1} \mathrm{H}-\mathrm{NMR}\left(300 \mathrm{MHz}, \mathrm{DMSO}-d_{6}\right): \delta 9.70$ (s, 1H, pyrazole-NH), $9.25(\mathrm{~s}, 1 \mathrm{H}, \mathrm{Ar}-\mathrm{NH}), 7.83(\mathrm{~d}, J=8.3 \mathrm{~Hz}, 1 \mathrm{H}, \mathrm{Ar}-\mathrm{H}), 7.52-7.49(\mathrm{~m}, 2 \mathrm{H}, \mathrm{Ar}-\mathrm{H})$, $3.80(\mathrm{t}, J=7.5 \mathrm{~Hz}, 1 \mathrm{H}, \mathrm{O}=\mathrm{C}-\mathrm{CH}-\mathrm{C}=\mathrm{O}), 3.62\left(\mathrm{~s}, 3 \mathrm{H}, \mathrm{N}-\mathrm{CH}_{3}\right), 2.94(\mathrm{t}, J=2.5 \mathrm{~Hz}, 1 \mathrm{H}, \mathrm{C} \equiv \mathrm{CH}), 2.84-2.80$ $\left(\mathrm{m}, 2 \mathrm{H}, \mathrm{CH}_{2}-\mathrm{C} \equiv \mathrm{CH}\right), 2.33\left(\mathrm{~s}, 3 \mathrm{H}, \mathrm{Ar}-\mathrm{CH}_{3}\right), 2.05\left(\mathrm{~s}, 3 \mathrm{H}\right.$, pyrazole- $\left.\mathrm{CH}_{3}\right), 1.96\left(\mathrm{~s}, 3 \mathrm{H}\right.$, pyrazole- $\left.\mathrm{CH}_{3}\right)$. ${ }^{13} \mathrm{C}-\mathrm{NMR}\left(75 \mathrm{MHz}, \mathrm{DMSO}-d_{6}\right): \delta 167.39(\mathrm{C}=\mathrm{O}), 166.93(\mathrm{C}=\mathrm{O}), 141.82\left(\mathrm{C}=\mathrm{N}\right.$, pyrazole- $\left.\mathrm{C}_{3}\right), 139.32$ (C-N-N, pyrazole-C $\left.\mathrm{C}_{5}\right), 134.23\left(\mathrm{C}-\mathrm{N}, \mathrm{Ar}-\mathrm{C}_{1}\right), 132.07\left(\mathrm{~d}, J_{\mathrm{CF}}=2.3 \mathrm{~Hz}, \mathrm{Ar}-\mathrm{C}_{2}\right), 127.24\left(\mathrm{~d}, J_{\mathrm{CF}}=9.8 \mathrm{~Hz}\right.$, Ar- $\left.C_{3}\right), 124.42\left(\mathrm{~d}, J_{\mathrm{CF}}=0.8 \mathrm{~Hz}, \operatorname{Ar}-\mathrm{C}_{6}\right), 123.52\left(\mathrm{~d}, J_{\mathrm{CF}}=9.8 \mathrm{~Hz}, \mathrm{Ar}-\mathrm{C}_{5}\right), 122.23\left(\mathrm{~d}, J_{\mathrm{CF}}=21 \mathrm{~Hz}\right.$, Ar- $\left.\mathrm{C}_{4}\right) 120.42\left(\mathrm{qd},{ }^{1} J_{\mathrm{CF}}=284.3 \mathrm{~Hz},{ }^{2} J_{\mathrm{CF}}=27.8 \mathrm{~Hz}, \mathrm{CF}_{3}\right), 114.80\left(\mathrm{C}-\mathrm{N}\right.$, pyrazole-C $\left.\mathrm{C}_{4}\right), 91.24$ (dsept, $\left.{ }^{1} J_{\mathrm{CF}}=200.3 \mathrm{~Hz},{ }^{2} J_{\mathrm{CF}}=33.0 \mathrm{~Hz}, \underline{\mathrm{CF}}\left(\mathrm{CF}_{3}\right)_{2}\right), 81.57(\underline{\mathrm{C}} \equiv \mathrm{CH}), 72.83(\mathrm{C} \equiv \underline{\mathrm{CH}}), 52.45(\mathrm{O}=\mathrm{C}-\underline{\mathrm{C}}-\mathrm{C}=\mathrm{O}), 36.08$ $\left(\mathrm{N}-\mathrm{C}\right.$, pyrazole- $\left.\mathrm{CH}_{3}\right), 19.33\left(\mathrm{CH}_{2}-\mathrm{C} \equiv \mathrm{CH}\right), 17.90\left(\mathrm{Ar}-\mathrm{CH}_{3}\right), 11.22$ (pyrazole- $\left.\mathrm{CH}_{3}\right), 9.10\left(\right.$ pyrazole- $\left.\mathrm{CH}_{3}\right)$. HRMS calcd. for $\mathrm{C}_{22} \mathrm{H}_{21} \mathrm{~F}_{7} \mathrm{~N}_{4} \mathrm{O}_{2}\left([\mathrm{M}+\mathrm{H}]^{+}\right)$, 507.1625; found, 507.1619.

2-Allyl-N $N^{1}$-(2-methyl-4-(perfluoropropan-2-yl)phenyl)-N ${ }^{3}-(1,3,5$-trimethyl-1H-pyrazol-4-yl)malonamide (8e): Light yellow solid; m.p. $155-157{ }^{\circ} \mathrm{C}$; yield $61.7 \% ;{ }^{1} \mathrm{H}-\mathrm{NMR}(300 \mathrm{MHz}$, Chloroform- $d): \delta 9.67(\mathrm{~s}, 1 \mathrm{H}$, pyrazole-NH), $8.38(\mathrm{~s}, 1 \mathrm{H}, \mathrm{Ar}-\mathrm{NH}), 8.13(\mathrm{~d}, J=9.3 \mathrm{~Hz}, 1 \mathrm{H}, \mathrm{Ar}-\mathrm{H}), 7.43-7.41(\mathrm{~m}, 2 \mathrm{H}, \mathrm{Ar}-\mathrm{H}), 5.96-5.78$ $\left(\mathrm{m}, 1 \mathrm{H}, \mathrm{CH}=\mathrm{CH}_{2}\right), 5.27-5.12\left(\mathrm{~m}, 2 \mathrm{H}, \mathrm{CH}=\mathrm{CH}_{2}\right), 3.68\left(\mathrm{~s}, 3 \mathrm{H}, \mathrm{N}-\mathrm{CH}_{3}\right), 3.54(\mathrm{t}, J=7.4 \mathrm{~Hz}, 1 \mathrm{H}$, $\mathrm{O}=\mathrm{C}-\mathrm{CH}-\mathrm{C}=\mathrm{O}), 3.42-3.33\left(\mathrm{~m}, 1 \mathrm{H}, \mathrm{O}=\mathrm{C}-\mathrm{CH}-\mathrm{CH}_{2}\right), 2.83\left(\mathrm{~s}, 3 \mathrm{H}, \mathrm{Ar}-\mathrm{CH}_{3}\right), 2.35\left(\mathrm{~s}, 3 \mathrm{H}\right.$, pyrazole- $\left.\mathrm{CH}_{3}\right)$, $2.09\left(\mathrm{~s}, 3 \mathrm{H}\right.$, pyrazole- $\left.\mathrm{CH}_{3}\right), 2.04-1.99\left(\mathrm{~m}, 1 \mathrm{H}, \mathrm{O}=\mathrm{C}-\mathrm{CH}-\mathrm{CH}_{2}\right) .{ }^{13} \mathrm{C}-\mathrm{NMR}(75 \mathrm{MHz}$, Chloroform- $d$ ): $\delta 170.56(\mathrm{C}=\mathrm{O}), 168.14(\mathrm{C}=\mathrm{O}), 142.68\left(\mathrm{C}=\mathrm{N}\right.$, pyrazole $\left.-\mathrm{C}_{3}\right), 137.83\left(\mathrm{C}-\mathrm{N}-\mathrm{N}\right.$, pyrazole $\left.-\mathrm{C}_{5}\right), 134.58$ $\left(\mathrm{C}-\mathrm{N}, \mathrm{Ar}-\mathrm{C}_{1}\right), 132.98\left(\underline{\mathrm{CH}}=\mathrm{CH}_{2}\right), 128.94\left(\mathrm{~d}, J_{\mathrm{CF}}=1.5 \mathrm{~Hz}, \mathrm{Ar}-\mathrm{C}_{2}\right), 127.33\left(\mathrm{~d}, J_{\mathrm{CF}}=10.5 \mathrm{~Hz}, \mathrm{Ar}-\mathrm{C}_{3}\right)$, $123.78\left(\mathrm{~d}, J_{\mathrm{CF}}=10.5 \mathrm{~Hz}, \mathrm{Ar}-\mathrm{C}_{5}\right), 122.33\left(\mathrm{~d}, J_{\mathrm{CF}}=20.3 \mathrm{~Hz}, \mathrm{Ar}-\mathrm{C}_{4}\right), 121.53\left(\mathrm{~d}, J_{\mathrm{CF}}=1.5 \mathrm{~Hz}, \mathrm{Ar}-\mathrm{C}_{6}\right)$, $115.71\left(\mathrm{qd},{ }^{1} J_{\mathrm{CF}}=284.3 \mathrm{~Hz},{ }^{2} J_{\mathrm{CF}}=27.0 \mathrm{~Hz}, \mathrm{CF}_{3}\right), 118.36\left(\mathrm{CH}=\mathrm{CH}_{2}\right), 113.51\left(\mathrm{C}-\mathrm{N}\right.$, pyrazole-C $\left.{ }_{4}\right), 90.96$ (dsept, $\left.{ }^{1} J_{\mathrm{CF}}=200.3 \mathrm{~Hz},{ }^{2} J_{\mathrm{CF}}=33.0 \mathrm{~Hz}, \underline{\mathrm{CF}}\left(\mathrm{CF}_{3}\right)_{2}\right), 54.39(\mathrm{O}=\mathrm{C}-\underline{\mathrm{C}}-\mathrm{C}=\mathrm{O}), 37.41\left(\mathrm{~N}-\mathrm{C}\right.$, pyrazole- $\left.\mathrm{CH}_{3}\right)$, 35.86 $\left(\mathrm{O}=\mathrm{C}-\mathrm{CH}-\mathrm{CH}_{2}\right), 17.71\left(\mathrm{Ar}-\mathrm{CH}_{3}\right), 10.90\left(\right.$ pyrazole- $\left.\mathrm{CH}_{3}\right), 9.08$ (pyrazole- $\left.\mathrm{CH}_{3}\right)$. HRMS calcd. for $\mathrm{C}_{22} \mathrm{H}_{23} \mathrm{~F}_{7} \mathrm{~N}_{4} \mathrm{O}_{2}\left([\mathrm{M}+\mathrm{H}]^{+}\right)$, 509.1782; found, 509.1777. 
$N^{1}$-(4-(1,1,1,3,3,3-Hexafluoro-2-methoxypropan-2-yl)-2-methylphenyl)-2-isopropyl- $N^{3}$-(1,3,5-trimethyl-1Hpyrazol-4-yl)malonamide (8f): Light yellow solid; m.p. $192-193{ }^{\circ} \mathrm{C}$; yield $58.4 \% ;{ }^{1} \mathrm{H}-\mathrm{NMR}(300 \mathrm{MHz}$, Chloroform- $d$ ): $\delta 9.50(\mathrm{~s}, 1 \mathrm{H}$, pyrazole-NH), $8.59(\mathrm{~s}, 1 \mathrm{H}, \mathrm{Ar}-\mathrm{NH}), 8.09(\mathrm{~d}, J=8.8 \mathrm{~Hz}, 1 \mathrm{H}, \mathrm{Ar}-\mathrm{H})$, 7.40-7.37 (m, 2H, Ar-H), $3.68\left(\mathrm{~s}, 3 \mathrm{H}, \mathrm{N}-\mathrm{CH}_{3}\right), 3.47\left(\mathrm{~s}, 3 \mathrm{H}, \mathrm{OCH}_{3}\right), 3.20(\mathrm{~d}, J=9.7 \mathrm{~Hz}, 1 \mathrm{H}, \mathrm{O}=\mathrm{C}-\mathrm{CH}-\mathrm{C}=\mathrm{O})$, 2.47-2.40 (m, $\left.1 \mathrm{H}, \mathrm{CH}\left(\mathrm{CH}_{3}\right)_{2}\right), 2.35\left(\mathrm{~s}, 3 \mathrm{H}, \mathrm{Ar}-\mathrm{CH}_{3}\right), 2.11\left(\mathrm{~s}, 6 \mathrm{H}\right.$, pyrazole- $\left.\mathrm{CH}_{3}\right), 1.13(\mathrm{dd}, J=8.5,6.7 \mathrm{~Hz}$, $\left.6 \mathrm{H}, \mathrm{CH}\left(\mathrm{CH}_{3}\right)_{2}\right) .{ }^{13} \mathrm{C}-\mathrm{NMR}(75 \mathrm{MHz}$, Chloroform- $d)$ : $\delta 170.71(\mathrm{C}=\mathrm{O}), 168.39(\mathrm{C}=\mathrm{O}), 142.68(\mathrm{C}=\mathrm{N}$, pyrazole- $\left.\mathrm{C}_{3}\right), 137.20\left(\mathrm{C}-\mathrm{N}-\mathrm{N}\right.$, pyrazole- $\left.\mathrm{C}_{5}\right), 134.48\left(\mathrm{C}-\mathrm{N}, \mathrm{Ar}-\mathrm{C}_{1}\right), 129.81\left(\mathrm{Ar}-\mathrm{C}_{2}\right), 128.54\left(\mathrm{Ar}-\mathrm{C}_{3}\right), 126.30$ $\left(\mathrm{Ar}-\mathrm{C}_{5}\right), 123.36\left(\mathrm{Ar}-\mathrm{C}_{4}\right), 122.06\left(\mathrm{q}, J_{\mathrm{CF}}=288.8 \mathrm{~Hz}, \mathrm{CF}_{3}\right), 121.26\left(\mathrm{Ar}^{-\mathrm{C}_{6}}\right), 113.73(\mathrm{C}-\mathrm{N}$, pyrazole-C 4$)$, 82.38 (hept, $\left.J_{\mathrm{CF}}=28.5 \mathrm{~Hz}, \mathrm{C}\left(\mathrm{CF}_{3}\right)_{2}\right), 62.65(\mathrm{O}=\mathrm{C}-\mathrm{C}-\mathrm{C}=\mathrm{O}), 53.83\left(\mathrm{OCH}_{3}\right), 35.89\left(\mathrm{~N}-\mathrm{C}\right.$, pyrazole- $\left.\mathrm{CH}_{3}\right)$, $32.70\left(\underline{\mathrm{CH}}\left(\mathrm{CH}_{3}\right)_{2}\right), 20.52\left(\mathrm{CH}\left(\mathrm{CH}_{3}\right)_{2}\right), 20.27\left(\mathrm{CH}\left(\mathrm{CH}_{3}\right)_{2}\right), 17.78\left(\mathrm{Ar}-\mathrm{CH}_{3}\right), 10.99$ (pyrazole- $\left.\mathrm{CH}_{3}\right), 9.15$ (pyrazole- $\left.\mathrm{CH}_{3}\right)$. HRMS calcd. for $\mathrm{C}_{23} \mathrm{H}_{28} \mathrm{~F}_{6} \mathrm{~N}_{4} \mathrm{O}_{3}\left([\mathrm{M}+\mathrm{H}]^{+}\right)$, 523.2138; found, 523.2132.

$N^{1}$-(4-(1,1,1,3,3,3-Hexafluoro-2-methoxypropan-2-yl)-2-methylphenyl)-2-isobutyl- $N^{3}-(1,3,5$-trimethyl-1Hpyrazol-4-yl)malonamide (8g): White solid; m.p. 204-205 ${ }^{\circ} \mathrm{C}$; yield $51.5 \% ;{ }^{1} \mathrm{H}-\mathrm{NMR}(300 \mathrm{MHz}$, Chloroform- $d$ ): $\delta 9.43(\mathrm{~s}, 1 \mathrm{H}$, pyrazole-NH), $8.47(\mathrm{~s}, 1 \mathrm{H}, \mathrm{Ar}-\mathrm{NH}), 8.06(\mathrm{~d}, J=9.2 \mathrm{~Hz}, 1 \mathrm{H}, \mathrm{Ar}-\mathrm{H})$, 7.40-7.38 (m, 2H, Ar-H), $3.69\left(\mathrm{~s}, 3 \mathrm{H}, \mathrm{N}-\mathrm{CH}_{3}\right), 3.64(\mathrm{t}, J=7.5 \mathrm{~Hz}, 1 \mathrm{H}, \mathrm{O}=\mathrm{C}-\mathrm{CH}-\mathrm{C}=\mathrm{O}), 3.47(\mathrm{~s}, 3 \mathrm{H}$, $\left.\mathrm{OCH}_{3}\right), 2.35\left(\mathrm{~s}, 3 \mathrm{H}, \mathrm{Ar}-\mathrm{CH}_{3}\right), 2.10\left(\mathrm{~s}, 6 \mathrm{H}\right.$, pyrazole- $\left.\mathrm{CH}_{3}\right), 2.03-1.88\left(\mathrm{~m}, 2 \mathrm{H}, \mathrm{O}=\mathrm{C}-\mathrm{CH}-\mathrm{CH}_{2}\right), 1.82-1.69$ $\left(\mathrm{m}, 1 \mathrm{H}, \mathrm{CH}\left(\mathrm{CH}_{3}\right)_{2}\right), 1.00\left(\mathrm{~d}, J=6.5 \mathrm{~Hz}, 6 \mathrm{H}, \mathrm{CH}\left(\mathrm{CH}_{3}\right)_{2}\right) .{ }^{13} \mathrm{C}-\mathrm{NMR}(75 \mathrm{MHz}$, Chloroform- $d$ ): $\delta 171.20$ $(\mathrm{C}=\mathrm{O}), 169.09(\mathrm{C}=\mathrm{O}), 142.65\left(\mathrm{C}=\mathrm{N}\right.$, pyrazole- $\left.\mathrm{C}_{3}\right), 137.13\left(\mathrm{C}-\mathrm{N}-\mathrm{N}\right.$, pyrazole- $\left.\mathrm{C}_{5}\right), 134.44\left(\mathrm{C}-\mathrm{N}, \mathrm{Ar}-\mathrm{C}_{1}\right)$, $129.80\left(\mathrm{Ar}^{-\mathrm{C}_{2}}\right), 128.63\left(\mathrm{Ar}^{-\mathrm{C}_{3}}\right), 126.30\left(\mathrm{Ar}-\mathrm{C}_{5}\right), 123.49\left(\mathrm{Ar}-\mathrm{C}_{4}\right), 122.06\left(\mathrm{q}, J_{\mathrm{CF}}=288.8 \mathrm{~Hz}, \mathrm{CF}_{3}\right)$, $121.39\left(\mathrm{Ar}-\mathrm{C}_{6}\right), 113.63\left(\mathrm{C}-\mathrm{N}\right.$, pyrazole- $\left.\mathrm{C}_{4}\right), 82.38$ (hept, $\left.J_{\mathrm{CF}}=28.5 \mathrm{~Hz}, \underline{\mathrm{C}}\left(\mathrm{CF}_{3}\right)_{2}\right), 53.84\left(\mathrm{OCH}_{3}\right), 53.53$ $(\mathrm{O}=\mathrm{C}-\mathrm{C}-\mathrm{C}=\mathrm{O}), 42.51\left(\mathrm{O}=\mathrm{C}-\mathrm{CH}-\mathrm{CH}_{2}\right), 35.90\left(\mathrm{~N}-\mathrm{C}\right.$, pyrazole- $\left.\mathrm{CH}_{3}\right), 26.18\left(\underline{\mathrm{CH}}\left(\mathrm{CH}_{3}\right)_{2}\right), 22.12\left(\mathrm{CH}\left(\underline{\mathrm{CH}}_{3}\right)_{2}\right)$, $21.73\left(\mathrm{CH}\left(\mathrm{CH}_{3}\right)_{2}\right), 17.75\left(\mathrm{Ar}-\mathrm{CH}_{3}\right), 10.89$ (pyrazole- $\left.\mathrm{CH}_{3}\right), 9.11$ (pyrazole- $\left.\mathrm{CH}_{3}\right)$. HRMS calcd. for $\mathrm{C}_{24} \mathrm{H}_{30} \mathrm{~F}_{6} \mathrm{~N}_{4} \mathrm{O}_{3}\left([\mathrm{M}+\mathrm{H}]^{+}\right)$, 537.2295; found, 537.2288.

$N^{1}$-(4-(1,1,1,3,3,3-Hexafluoro-2-methoxypropan-2-yl)-2-methylphenyl)-2-isopentyl- $N^{3}$-(1,3,5-trimethyl-1Hpyrazol-4-yl)malonamide (8h): White solid; m.p. 186-187 ${ }^{\circ} \mathrm{C}$; yield $56.1 \%$; ${ }^{1} \mathrm{H}-\mathrm{NMR}(300 \mathrm{MHz}$, Chloroform- $d$ ): $\delta 9.55(\mathrm{~s}, 1 \mathrm{H}$, pyrazole-NH), $8.40(\mathrm{~s}, 1 \mathrm{H}, \mathrm{Ar}-\mathrm{NH}), 8.10(\mathrm{~d}, J=8.4 \mathrm{~Hz}, 1 \mathrm{H}, \mathrm{Ar}-\mathrm{H}), 7.41-7.38$ $(\mathrm{m}, 2 \mathrm{H}, \mathrm{Ar}-\mathrm{H}), 3.69\left(\mathrm{~s}, 3 \mathrm{H}, \mathrm{N}-\mathrm{CH}_{3}\right), 3.47\left(\mathrm{~s}, 3 \mathrm{H}, \mathrm{OCH}_{3}\right), 3.45(\mathrm{t}, \mathrm{J}=6.0 \mathrm{~Hz}, 1 \mathrm{H}, \mathrm{O}=\mathrm{C}-\mathrm{CH}-\mathrm{C}=\mathrm{O}), 2.35$ $\left(\mathrm{s}, 3 \mathrm{H}, \mathrm{Ar}-\mathrm{CH}_{3}\right), 2.12\left(\mathrm{~s}, 6 \mathrm{H}\right.$, pyrazole- $\left.\mathrm{CH}_{3}\right), 2.10-2.03\left(\mathrm{~m}, 2 \mathrm{H}, \mathrm{O}=\mathrm{C}-\mathrm{CH}-\mathrm{CH}_{2}\right), 1.65-1.56(\mathrm{~m}, 1 \mathrm{H}$, $\left.\mathrm{CH}\left(\mathrm{CH}_{3}\right)_{2}\right), 1.41-1.33\left(\mathrm{~m}, 2 \mathrm{H}, \mathrm{CH}_{2}-\mathrm{CH}\left(\mathrm{CH}_{3}\right)_{2}\right), 0.88\left(\mathrm{~d}, \mathrm{~J}=6.6 \mathrm{~Hz}, 6 \mathrm{H}, \mathrm{CH}\left(\mathrm{CH}_{3}\right)_{2}\right) .{ }^{13} \mathrm{C}-\mathrm{NMR}$ (75 MHz, Chloroform- $d$ ): $\delta 171.30(\mathrm{C}=\mathrm{O}), 169.01(\mathrm{C}=\mathrm{O}), 142.63\left(\mathrm{C}=\mathrm{N}\right.$, pyrazole- $\left.\mathrm{C}_{3}\right), 137.17(\mathrm{C}-\mathrm{N}-\mathrm{N}$, pyrazole- $\left.\mathrm{C}_{5}\right), 134.45\left(\mathrm{C}-\mathrm{N}, \mathrm{Ar}-\mathrm{C}_{1}\right), 129.81\left(\mathrm{Ar}-\mathrm{C}_{2}\right), 128.64\left(\mathrm{Ar}-\mathrm{C}_{3}\right), 126.28\left(\mathrm{Ar}-\mathrm{C}_{5}\right), 123.45\left(\mathrm{Ar}-\mathrm{C}_{4}\right)$, $122.06\left(\mathrm{q}, J_{\mathrm{CF}}=288.8 \mathrm{~Hz}, \mathrm{CF}_{3}\right), 121.38\left(\mathrm{Ar}-\mathrm{C}_{6}\right), 113.68\left(\mathrm{C}-\mathrm{N}\right.$, pyrazole- $\left.\mathrm{C}_{4}\right), 82.38\left(\mathrm{hept}, J_{\mathrm{CF}}=28.5 \mathrm{~Hz}\right.$, $\left.\underline{\mathrm{C}}\left(\mathrm{CF}_{3}\right)_{2}\right), 55.06(\mathrm{O}=\mathrm{C}-\underline{\mathrm{C}}-\mathrm{C}=\mathrm{O}), 53.81\left(\mathrm{OCH}_{3}\right), 36.30\left(\mathrm{~N}-\mathrm{C}\right.$, pyrazole- $\left.\mathrm{CH}_{3}\right), 35.86\left(\mathrm{C}_{2}-\mathrm{CH}\left(\mathrm{CH}_{3}\right)_{2}\right)$, $31.62\left(\underline{\mathrm{CH}}\left(\mathrm{CH}_{3}\right)_{2}\right), 27.37\left(\mathrm{O}=\mathrm{C}-\mathrm{CH}-\mathrm{CH}_{2}\right), 21.96\left(\mathrm{CH}\left(\mathrm{CH}_{3}\right)_{2}\right), 21.88\left(\mathrm{CH}\left(\mathrm{CH}_{3}\right)_{2}\right), 17.78\left(\mathrm{Ar}-\mathrm{CH}_{3}\right)$, 10.92 (pyrazole- $\left.\mathrm{CH}_{3}\right), 9.10$ (pyrazole- $\left.\mathrm{CH}_{3}\right)$. HRMS calcd. for $\mathrm{C}_{25} \mathrm{H}_{32} \mathrm{~F}_{6} \mathrm{~N}_{4} \mathrm{O}_{3}\left([\mathrm{M}+\mathrm{H}]^{+}\right), 551.2451$; found, 551.2446 .

$N^{1}$-(4-(1,1,1,3,3,3-Hexafluoro-2-methoxypropan-2-yl)-2-methylphenyl)-2-(prop-2-yn-1-yl)- $N^{3}-(1,3,5$-trimethyl1H-pyrazol-4-yl)malonamide (8i): Light yellow solid; m.p. $54-55{ }^{\circ} \mathrm{C}$; yield $46.7 \%$; ${ }^{1} \mathrm{H}-\mathrm{NMR}(300 \mathrm{MHz}$, Chloroform-d): $\delta 9.61(\mathrm{~s}, 1 \mathrm{H}$, pyrazole-NH), $8.48(\mathrm{~s}, 1 \mathrm{H}, \mathrm{Ar}-\mathrm{NH}), 8.05(\mathrm{~d}, J=8.5 \mathrm{~Hz}, 1 \mathrm{H}, \mathrm{Ar}-\mathrm{H}), 7.40-7.37$ $(\mathrm{m}, 2 \mathrm{H}, \mathrm{Ar}-\mathrm{H}), 3.69-3.64\left(\mathrm{~m}, 4 \mathrm{H}, \mathrm{O}=\mathrm{C}-\mathrm{CH}-\mathrm{C}=\mathrm{O}\right.$ and $\left.\mathrm{N}-\mathrm{CH}_{3}\right), 3.46\left(\mathrm{~s}, 3 \mathrm{H}, \mathrm{OCH}_{3}\right), 2.97(\mathrm{dd}, J=7.5$, $\left.2.5 \mathrm{~Hz}, 2 \mathrm{H}, \mathrm{CH}_{2}-\mathrm{C} \equiv \mathrm{CH}\right), 2.33\left(\mathrm{~s}, 3 \mathrm{H}, \mathrm{Ar}-\mathrm{CH}_{3}\right), 2.15(\mathrm{t}, J=2.6 \mathrm{~Hz}, 1 \mathrm{H}, \mathrm{C} \equiv \mathrm{CH}), 2.11(\mathrm{~d}, J=2.9 \mathrm{~Hz}$, 6H, pyrazole- $\left.\mathrm{CH}_{3}\right) .{ }^{13} \mathrm{C}-\mathrm{NMR}(75 \mathrm{MHz}$, Chloroform-d): $\delta 169.54(\mathrm{C}=\mathrm{O}), 166.75(\mathrm{C}=\mathrm{O}), 142.79(\mathrm{C}=\mathrm{N}$, pyrazole- $\left.\mathrm{C}_{3}\right), 136.94\left(\mathrm{C}-\mathrm{N}-\mathrm{N}\right.$, pyrazole- $\left.\mathrm{C}_{5}\right), 134.78\left(\mathrm{C}-\mathrm{N}, \mathrm{Ar}-\mathrm{C}_{1}\right), 129.80\left(\mathrm{Ar}-\mathrm{C}_{2}\right), 129.09\left(\mathrm{Ar}-\mathrm{C}_{3}\right), 126.27$ $\left(\right.$ Ar- $\left.\mathrm{C}_{5}\right), 123.77\left(\mathrm{Ar}-\mathrm{C}_{4}\right), 122.04\left(\mathrm{q}, J_{\mathrm{CF}}=288.8 \mathrm{~Hz}, \mathrm{CF}_{3}\right), 121.79\left(\mathrm{Ar}-\mathrm{C}_{6}\right), 113.47\left(\mathrm{C}-\mathrm{N}\right.$, pyrazole- $\left.\mathrm{C}_{4}\right), 82.36$ (hept, $\left.J_{\mathrm{CF}}=28.5 \mathrm{~Hz}, \underline{\mathrm{C}}\left(\mathrm{CF}_{3}\right)_{2}\right), 79.41(\underline{\mathrm{C}} \equiv \mathrm{CH}), 71.64(\mathrm{C} \equiv \underline{\mathrm{CH}}), 53.83(\mathrm{O}=\mathrm{C}-\underline{\mathrm{C}}-\mathrm{C}=\mathrm{O}), 52.84\left(\mathrm{OCH}_{3}\right), 35.83$ $\left(\mathrm{N}-\mathrm{C}\right.$, pyrazole- $\left.\mathrm{CH}_{3}\right), 22.06\left(\mathrm{CH}_{2}-\mathrm{C} \equiv \mathrm{CH}\right), 17.78\left(\mathrm{Ar}^{-} \mathrm{CH}_{3}\right), 10.86$ (pyrazole- $\left.\mathrm{CH}_{3}\right), 9.03$ (pyrazole- $\left.\mathrm{CH}_{3}\right)$. HRMS calcd. for $\mathrm{C}_{23} \mathrm{H}_{24} \mathrm{~F}_{6} \mathrm{~N}_{4} \mathrm{O}_{3}\left([\mathrm{M}+\mathrm{H}]^{+}\right)$, 519.1825; found, 519.1818. 
2-Allyl-N $N^{1}-(4-(1,1,1,3,3,3-h e x a f l u o r o-2-m e t h o x y p r o p a n-2-y l)-2-m e t h y l p h e n y l)-N^{3}-(1,3,5-t r i m e t h y l-1 H-p y r a z o l-$ 4-yl)malonamide (8j): White solid; m.p. $146-148{ }^{\circ} \mathrm{C}$; yield 62.5\%; ${ }^{1} \mathrm{H}-\mathrm{NMR}(300 \mathrm{MHz}$, Chloroform- $d): \delta$ $9.56(\mathrm{~s}, 1 \mathrm{H}$, pyrazole-NH), 8.30 (s, 1H, Ar-NH), 8.07 (d, J = $8.7 \mathrm{~Hz}, 1 \mathrm{H}, \mathrm{Ar}-\mathrm{H}), 7.40-7.38(\mathrm{~m}, 2 \mathrm{H}, \mathrm{Ar}-\mathrm{H})$, 5.93-5.79 (m, $\left.1 \mathrm{H}, \mathrm{CH}=\mathrm{CH}_{2}\right), 5.28-5.12\left(\mathrm{~m}, 2 \mathrm{H}, \mathrm{CH}=\mathrm{CH}_{2}\right), 3.68\left(\mathrm{~s}, 3 \mathrm{H}, \mathrm{N}-\mathrm{CH}_{3}\right), 3.55(\mathrm{t}, J=7.4 \mathrm{~Hz}, 1 \mathrm{H}$, $\mathrm{O}=\mathrm{C}-\mathrm{CH}-\mathrm{C}=\mathrm{O}), 3.47\left(\mathrm{~s}, 3 \mathrm{H}, \mathrm{OCH}_{3}\right), 2.82\left(\mathrm{t}, J=7.2 \mathrm{~Hz}, 2 \mathrm{H}, \mathrm{O}=\mathrm{C}-\mathrm{CH}-\mathrm{CH}_{2}\right), 2.34\left(\mathrm{~s}, 3 \mathrm{H}, \mathrm{Ar}^{-\mathrm{CH}_{3}}\right), 2.09$ $\left(\mathrm{d}, J=1.4 \mathrm{~Hz}, 6 \mathrm{H}\right.$, pyrazole- $\left.\mathrm{CH}_{3}\right) .{ }^{13} \mathrm{C}-\mathrm{NMR}(75 \mathrm{MHz}$, Chloroform- $d): \delta 170.56(\mathrm{C}=\mathrm{O}), 168.07(\mathrm{C}=\mathrm{O})$,

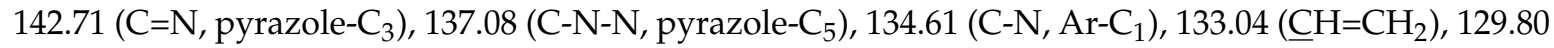
$\left(\mathrm{Ar}-\mathrm{C}_{2}\right), 128.73\left(\mathrm{Ar}-\mathrm{C}_{3}\right), 126.31\left(\mathrm{Ar}-\mathrm{C}_{5}\right), 123.54\left(\mathrm{Ar}-\mathrm{C}_{4}\right), 122.05\left(\mathrm{q}, J_{\mathrm{CF}}=288.8 \mathrm{~Hz}, \mathrm{CF}_{3}\right), 121.49\left(\mathrm{Ar}-\mathrm{C}_{6}\right)$, $118.35\left(\mathrm{CH}=\mathrm{CH}_{2}\right), 113.50\left(\mathrm{C}-\mathrm{N}\right.$, pyrazole- $\left.\mathrm{C}_{4}\right), 82.38$ (hept, $\left.J_{\mathrm{CF}}=28.5 \mathrm{~Hz}, \underline{\mathrm{C}}\left(\mathrm{CF}_{3}\right)_{2}\right), 54.41(\mathrm{O}=\mathrm{C}-\mathrm{C}-\mathrm{C}=\mathrm{O})$, $53.84\left(\mathrm{OCH}_{3}\right), 37.40\left(\mathrm{~N}-\mathrm{C}\right.$, pyrazole- $\left.\mathrm{CH}_{3}\right), 35.88\left(\mathrm{O}=\mathrm{C}-\mathrm{CH}-\mathrm{CH}_{2}\right), 17.81\left(\mathrm{Ar}^{\left.-\mathrm{CH}_{3}\right)}\right), 10.90\left(\right.$ pyrazole- $\left.\mathrm{CH}_{3}\right)$, 9.11 (pyrazole- $\mathrm{CH}_{3}$ ). HRMS calcd. for $\mathrm{C}_{23} \mathrm{H}_{26} \mathrm{~F}_{6} \mathrm{~N}_{4} \mathrm{O}_{3}\left([\mathrm{M}+\mathrm{H}]^{+}\right.$), 521.1982; found, 521.1976.

$N^{1}$-(4-(1,1,1,3,3,3-Hexafluoro-2-methoxypropan-2-yl)-2-methoxyphenyl)-2-isopropyl- $N^{3}$-(1,3,5-trimethyl-1Hpyrazol-4-yl)malonamide (8k): Light yellow solid; m.p. $74-76{ }^{\circ} \mathrm{C}$; yield $58.3 \%$; ${ }^{1} \mathrm{H}-\mathrm{NMR}(300 \mathrm{MHz}$, Chloroform-d): $\delta 9.39$ (s, 1H, pyrazole-NH), 8.42 (d, $J=8.6 \mathrm{~Hz}, 1 \mathrm{H}, \mathrm{Ar}-\mathrm{H}), 8.37$ (s, 1H, Ar-NH), $7.16(\mathrm{~d}, J=8.6 \mathrm{~Hz}, 1 \mathrm{H}, \mathrm{Ar}-\mathrm{H}), 7.05(\mathrm{~s}, 1 \mathrm{H}, \mathrm{Ar}-\mathrm{H}), 3.91\left(\mathrm{~s}, 3 \mathrm{H}, \mathrm{Ar}-\mathrm{OCH}_{3}\right), 3.68\left(\mathrm{~s}, 3 \mathrm{H}, \mathrm{N}-\mathrm{CH}_{3}\right), 3.49$ $\left(\mathrm{s}, 3 \mathrm{H}, \mathrm{OCH}_{3}\right), 3.09(\mathrm{~d}, J=9.9 \mathrm{~Hz}, 1 \mathrm{H}, \mathrm{O}=\mathrm{C}-\mathrm{CH}-\mathrm{C}=\mathrm{O}), 2.51-2.40\left(\mathrm{~m}, 1 \mathrm{H}, \mathrm{CH}\left(\mathrm{CH}_{3}\right)_{2}\right), 2.11(\mathrm{~s}, 6 \mathrm{H}$, pyrazole- $\left.\mathrm{CH}_{3}\right), 1.11\left(\mathrm{dd}, J=16.9,6.6 \mathrm{~Hz}, 6 \mathrm{H}, \mathrm{CH}\left(\mathrm{CH}_{3}\right)_{2}\right) \cdot{ }^{13} \mathrm{C}-\mathrm{NMR}(75 \mathrm{MHz}$, Chloroform-d): $\delta 169.60$ $(\mathrm{C}=\mathrm{O}), 168.48(\mathrm{C}=\mathrm{O}), 148.25\left(\right.$ Ar- $\left._{2}\right), 142.72\left(\mathrm{C}=\mathrm{N}\right.$, pyrazole- $\left.\mathrm{C}_{3}\right), 134.46\left(\mathrm{C}-\mathrm{N}, \mathrm{Ar}-\mathrm{C}_{1}\right), 128.74(\mathrm{C}-\mathrm{N}-\mathrm{N}$, pyrazole- $\left.\mathrm{C}_{5}\right), 122.90\left(\mathrm{Ar}-\mathrm{C}_{4}\right), 122.05\left(\mathrm{q}, J_{\mathrm{CF}}=288.8 \mathrm{~Hz}, \mathrm{CF}_{3}\right), 120.76\left(\mathrm{Ar}-\mathrm{C}_{5}\right), 119.24\left(\mathrm{Ar}-\mathrm{C}_{6}\right), 113.84$ $\left(\mathrm{C}-\mathrm{N}\right.$, pyrazole- $\left.\mathrm{C}_{4}\right), 109.51\left(\mathrm{Ar}-\mathrm{C}_{3}\right), 82.45$ (hept, $\left.J_{\mathrm{CF}}=28.5 \mathrm{~Hz}, \underline{\mathrm{C}}\left(\mathrm{CF}_{3}\right)_{2}\right), 63.58(\mathrm{O}=\mathrm{C}-\mathrm{C}-\mathrm{C}=\mathrm{O}), 55.64$ $\left(\mathrm{Ar}-\mathrm{OCH}_{3}\right), 53.90\left(\mathrm{OCH}_{3}\right), 35.87\left(\mathrm{~N}-\mathrm{C}\right.$, pyrazole- $\left.\mathrm{CH}_{3}\right), 32.35\left(\mathrm{CH}\left(\mathrm{CH}_{3}\right)_{2}\right), 20.45\left(\mathrm{CH}\left(\mathrm{CH}_{3}\right)_{2}\right), 20.24$ $\left(\mathrm{CH}\left(\mathrm{CH}_{3}\right)_{2}\right), 10.95$ (pyrazole- $\left.\mathrm{CH}_{3}\right), 9.19$ (pyrazole- $\left.\mathrm{CH}_{3}\right)$. HRMS calcd. for $\mathrm{C}_{23} \mathrm{H}_{28} \mathrm{~F}_{6} \mathrm{~N}_{4} \mathrm{O}_{4}\left([\mathrm{M}+\mathrm{H}]^{+}\right)$, 539.2088; found, 539.2081.

$N^{1}$-(4-(1,1,1,3,3,3-Hexafluoro-2-methoxypropan-2-yl)-2-methoxyphenyl)-2-isobutyl-N ${ }^{3}$-(1,3,5-trimethyl-1Hpyrazol-4-yl)malonamide (81): White solid; m.p. $193-195{ }^{\circ} \mathrm{C}$; yield $51.8 \%$; ${ }^{1} \mathrm{H}-\mathrm{NMR}(300 \mathrm{MHz}$, Chloroform-d): $\delta 9.32$ (s, 1H, pyrazole-NH), 8.41 (d, J = $8.6 \mathrm{~Hz}, 1 \mathrm{H}, \mathrm{Ar}-\mathrm{H}), 8.29$ (s, 1H, Ar-NH), $7.16(\mathrm{~d}, J=8.6 \mathrm{~Hz}, 1 \mathrm{H}, \mathrm{Ar}-\mathrm{H}), 7.05(\mathrm{~s}, 1 \mathrm{H}, \mathrm{Ar}-\mathrm{H}), 3.92\left(\mathrm{~s}, 3 \mathrm{H}, \mathrm{Ar}-\mathrm{OCH}_{3}\right), 3.68\left(\mathrm{~s}, 3 \mathrm{H}, \mathrm{N}-\mathrm{CH}_{3}\right), 3.55$ $(\mathrm{t}, J=7.6 \mathrm{~Hz}, 1 \mathrm{H}, \mathrm{O}=\mathrm{C}-\mathrm{CH}-\mathrm{C}=\mathrm{O}), 3.49\left(\mathrm{~s}, 3 \mathrm{H}, \mathrm{OCH}_{3}\right), 2.10\left(\mathrm{~d}, J=1.1 \mathrm{~Hz}, 6 \mathrm{H}\right.$, pyrazole- $\left.\mathrm{CH}_{3}\right)$, 2.04-1.84 (m, 2H, O=C-CH-CH $)_{2}, 1.80-1.67\left(\mathrm{~m}, 1 \mathrm{H}, \mathrm{CH}\left(\mathrm{CH}_{3}\right)_{2}\right), 0.99\left(\mathrm{~d}, J=6.5 \mathrm{~Hz}, 6 \mathrm{H}, \mathrm{CH}\left(\mathrm{CH}_{3}\right)_{2}\right)$. ${ }^{13} \mathrm{C}-\mathrm{NMR}\left(75 \mathrm{MHz}\right.$, Chloroform-d): $\delta 170.02(\mathrm{C}=\mathrm{O}), 169.05(\mathrm{C}=\mathrm{O}), 148.18\left(\mathrm{Ar}-\mathrm{C}_{2}\right), 142.72(\mathrm{C}=\mathrm{N}$, pyrazole- $\left.\mathrm{C}_{3}\right), 134.47\left(\mathrm{C}-\mathrm{N}, \mathrm{Ar}-\mathrm{C}_{1}\right), 128.70\left(\mathrm{C}-\mathrm{N}-\mathrm{N}\right.$, pyrazole- $\left.\mathrm{C}_{5}\right), 122.98\left(\mathrm{Ar}-\mathrm{C}_{4}\right), 120.78\left(\mathrm{Ar}-\mathrm{C}_{5}\right)$, $122.04\left(\mathrm{q}, J_{\mathrm{CF}}=288.8 \mathrm{~Hz}, \mathrm{CF}_{3}\right), 119.27\left(\mathrm{Ar}-\mathrm{C}_{6}\right), 113.72\left(\mathrm{C}-\mathrm{N}\right.$, pyrazole- $\left.\mathrm{C}_{4}\right), 109.46\left(\mathrm{Ar}-\mathrm{C}_{3}\right), 82.45$ (hept, $\left.J_{\mathrm{CF}}=28.5 \mathrm{~Hz}, \underline{\mathrm{C}}\left(\mathrm{CF}_{3}\right)_{2}\right), 55.64\left(\mathrm{Ar}-\mathrm{OCH}_{3}\right), 54.35\left(\mathrm{OCH}_{3}\right), 53.92(\mathrm{O}=\mathrm{C}-\mathrm{C}-\mathrm{C}=\mathrm{O}), 41.75\left(\mathrm{O}=\mathrm{C}-\mathrm{CH}-\underline{C H}_{2}\right)$, $35.90\left(\mathrm{~N}-\mathrm{C}\right.$, pyrazole- $\left.\mathrm{CH}_{3}\right), 26.16\left(\mathrm{CH}\left(\mathrm{CH}_{3}\right)_{2}\right), 22.11\left(\mathrm{CH}\left(\mathrm{CH}_{3}\right)_{2}\right), 21.78\left(\mathrm{CH}\left(\mathrm{CH}_{3}\right)_{2}\right), 10.85$ (pyrazole- $\mathrm{CH}_{3}$ ), 9.15 (pyrazole- $\mathrm{CH}_{3}$ ). HRMS calcd. for $\mathrm{C}_{24} \mathrm{H}_{30} \mathrm{~F}_{6} \mathrm{~N}_{4} \mathrm{O}_{4}\left([\mathrm{M}+\mathrm{H}]^{+}\right), 553.2244$; found, 553.2236 .

$N^{1}$-(4-(1,1,1,3,3,3-Hexafluoro-2-methoxypropan-2-yl)-2-methoxyphenyl)-2-isopentyl- $N^{3}$-(1,3,5-trimethyl-1Hpyrazol-4-yl)malonamide (8m): Light yellow solid; m.p. $70-72{ }^{\circ} \mathrm{C}$; yield $47.2 \% ;{ }^{1} \mathrm{H}-\mathrm{NMR}(300 \mathrm{MHz}$, Chloroform-d): $\delta 9.32(\mathrm{~s}, 1 \mathrm{H}$, pyrazole-NH), $8.43(\mathrm{~d}, J=8.6 \mathrm{~Hz}, 1 \mathrm{H}, \mathrm{Ar}-\mathrm{H}), 8.17(\mathrm{~s}, 1 \mathrm{H}, \mathrm{Ar}-\mathrm{NH})$, $7.17(\mathrm{~d}, J=8.4 \mathrm{~Hz}, 1 \mathrm{H}, \mathrm{Ar}-\mathrm{H}), 7.06(\mathrm{~s}, 1 \mathrm{H}, \mathrm{Ar}-\mathrm{H}), 3.92\left(\mathrm{~s}, 3 \mathrm{H}, \mathrm{Ar}-\mathrm{OCH}_{3}\right), 3.69\left(\mathrm{~s}, 3 \mathrm{H}, \mathrm{N}-\mathrm{CH}_{3}\right), 3.49$ $\left(\mathrm{s}, 3 \mathrm{H}, \mathrm{OCH}_{3}\right), 3.36(\mathrm{t}, J=7.5 \mathrm{~Hz}, 1 \mathrm{H}, \mathrm{O}=\mathrm{C}-\mathrm{CH}-\mathrm{C}=\mathrm{O}), 2.12\left(\mathrm{~s}, 6 \mathrm{H}\right.$, pyrazole- $\left.\mathrm{CH}_{3}\right), 2.09-2.03(\mathrm{~m}, 2 \mathrm{H}$, $\left.\mathrm{O}=\mathrm{C}-\mathrm{CH}-\mathrm{CH}_{2}\right), 1.67-1.54\left(\mathrm{~m}, 1 \mathrm{H}, \mathrm{CH}\left(\mathrm{CH}_{3}\right)_{2}\right), 1.40-1.31\left(\mathrm{~m}, 2 \mathrm{H}, \mathrm{CH}_{2}-\mathrm{CH}\left(\mathrm{CH}_{3}\right)_{2}\right), 0.88(\mathrm{~d}, J=6.6 \mathrm{~Hz}$, $\left.6 \mathrm{H}, \mathrm{CH}\left(\mathrm{CH}_{3}\right)_{2}\right) .{ }^{13} \mathrm{C}-\mathrm{NMR}\left(75 \mathrm{MHz}\right.$, Chloroform-d): $\delta 170.05(\mathrm{C}=\mathrm{O}), 169.01(\mathrm{C}=\mathrm{O}), 148.23\left(\mathrm{Ar}-\mathrm{C}_{2}\right)$, $142.71\left(\mathrm{C}=\mathrm{N}\right.$, pyrazole- $\left.\mathrm{C}_{3}\right), 134.47\left(\mathrm{C}-\mathrm{N}, \mathrm{Ar}-\mathrm{C}_{1}\right), 128.71\left(\mathrm{C}-\mathrm{N}-\mathrm{N}\right.$, pyrazole- $\left.\mathrm{C}_{5}\right), 122.98\left(\mathrm{Ar}^{\left.-\mathrm{C}_{4}\right)}, 120.78\right.$ $\left(\mathrm{Ar}-\mathrm{C}_{5}\right), 122.04\left(\mathrm{q}, J_{\mathrm{CF}}=288.8 \mathrm{~Hz}, \mathrm{CF}_{3}\right), 119.34\left(\mathrm{Ar}-\mathrm{C}_{6}\right), 113.74\left(\mathrm{C}-\mathrm{N}\right.$, pyrazole- $\left.\mathrm{C}_{4}\right), 109.48\left(\mathrm{Ar}-\mathrm{C}_{3}\right)$, 82.45 (hept, $\left.J_{\mathrm{CF}}=28.5 \mathrm{~Hz}, \underline{\mathrm{C}}\left(\mathrm{CF}_{3}\right)_{2}\right), 56.01\left(\mathrm{Ar}-\mathrm{OCH}_{3}\right), 55.63(\mathrm{O}=\mathrm{C}-\mathrm{C}-\mathrm{C}=\mathrm{O}), 53.90\left(\mathrm{OCH}_{3}\right), 36.27(\mathrm{~N}-\mathrm{C}$, pyrazole- $\left.\mathrm{CH}_{3}\right), 35.88\left(\mathrm{CH}_{2}-\mathrm{CH}\left(\mathrm{CH}_{3}\right)_{2}\right), 31.01\left(\underline{\mathrm{CH}}\left(\mathrm{CH}_{3}\right)_{2}\right), 27.43\left(\mathrm{O}=\mathrm{C}-\mathrm{CH}-\underline{\mathrm{C}} \mathrm{H}_{2}\right), 22.01\left(\mathrm{CH}\left(\mathrm{CH}_{3}\right)_{2}\right)$, $21.92\left(\mathrm{CH}\left(\mathrm{CH}_{3}\right)_{2}\right), 10.88$ (pyrazole- $\left.\mathrm{CH}_{3}\right), 9.16$ (pyrazole- $\left.\mathrm{CH}_{3}\right)$. HRMS calcd. for $\mathrm{C}_{25} \mathrm{H}_{32} \mathrm{~F}_{6} \mathrm{~N}_{4} \mathrm{O}_{4}$ $\left([\mathrm{M}+\mathrm{H}]^{+}\right), 567.2401$; found, 567.2391. 
$N^{1}$-(4-(1,1,1,3,3,3-Hexafluoro-2-methoxypropan-2-yl)-2-methoxyphenyl)-2-(prop-2-yn-1-yl)-N $N^{3}-(1,3,5$-trimethyl1H-pyrazol-4-yl)malonamide (8n): Light yellow solid; m.p. $178-180{ }^{\circ} \mathrm{C}$; yield $60.2 \%$; ${ }^{1} \mathrm{H}-\mathrm{NMR}(300 \mathrm{MHz}$, DMSO-d $\left._{6}\right): \delta 9.64(\mathrm{~s}, 1 \mathrm{H}$, pyrazole-NH) $9.37(\mathrm{~s}, 1 \mathrm{H}, \mathrm{Ar}-\mathrm{NH}), 8.31(\mathrm{~d}, J=8.6 \mathrm{~Hz}, 1 \mathrm{H}, \mathrm{Ar}-\mathrm{H})$, $7.17(\mathrm{~d}, J=8.7 \mathrm{~Hz}, 1 \mathrm{H}, \mathrm{Ar}-\mathrm{H}), 7.08(\mathrm{~s}, 1 \mathrm{H}, \mathrm{Ar}-\mathrm{H}), 3.92\left(\mathrm{~s}, 3 \mathrm{H}, \mathrm{Ar}-\mathrm{OCH}_{3}\right), 3.86(\mathrm{t}, J=7.6 \mathrm{~Hz}, 1 \mathrm{H}$, $\mathrm{O}=\mathrm{C}-\mathrm{CH}-\mathrm{C}=\mathrm{O}), 3.62\left(\mathrm{~s}, 3 \mathrm{H}, \mathrm{N}-\mathrm{CH}_{3}\right), 3.48\left(\mathrm{~s}, 3 \mathrm{H}, \mathrm{OCH}_{3}\right), 2.93(\mathrm{t}, J=2.5 \mathrm{~Hz}, 1 \mathrm{H}, \mathrm{C} \equiv \mathrm{CH}), 2.78-2.74$ $\left(\mathrm{m}, 2 \mathrm{H}, \mathrm{CH}_{2}-\mathrm{C} \equiv \mathrm{CH}\right), 2.05\left(\mathrm{~s}, 3 \mathrm{H}\right.$, pyrazole- $\left.\mathrm{CH}_{3}\right), 1.96\left(\mathrm{~s}, 3 \mathrm{H}\right.$, pyrazole- $\left.\mathrm{CH}_{3}\right) .{ }^{13} \mathrm{C}-\mathrm{NMR}(75 \mathrm{MHz}$, DMSO- $\left._{6}\right): \delta 167.45(\mathrm{C}=\mathrm{O}), 166.67(\mathrm{C}=\mathrm{O}), 148.93\left(\right.$ Ar- $\left.\mathrm{C}_{2}\right), 141.74\left(\mathrm{C}=\mathrm{N}\right.$, pyrazole- $\left.\mathrm{C}_{3}\right), 134.18$ $\left(\mathrm{C}-\mathrm{N}, \mathrm{Ar}-\mathrm{C}_{1}\right), 129.47\left(\mathrm{C}-\mathrm{N}-\mathrm{N}\right.$, pyrazole- $\left.\mathrm{C}_{5}\right), 122.35$ (q, J $\left.J_{\mathrm{CF}}=288.8 \mathrm{~Hz}, \mathrm{CF}_{3}\right), 121.93\left(\mathrm{Ar}-\mathrm{C}_{4}\right), 120.64$ $\left(\right.$ Ar- $\left.C_{5}\right), 120.51\left(\right.$ Ar- $\left.C_{6}\right), 114.70\left(\mathrm{C}-\mathrm{N}\right.$, pyrazole-C $\left.{ }_{4}\right), 110.17\left(\mathrm{Ar}_{-} \mathrm{C}_{3}\right), 82.47$ (hept, $J_{\mathrm{CF}}=28.5 \mathrm{~Hz}$, $\left.\underline{\mathrm{C}}\left(\mathrm{CF}_{3}\right)_{2}\right), 81.48(\underline{\mathrm{C}} \equiv \mathrm{CH}), 72.89(\mathrm{C} \equiv \mathrm{CH}), 56.28\left(\mathrm{Ar}^{-O C H_{3}}\right), 54.48(\mathrm{O}=\mathrm{C}-\mathrm{C}-\mathrm{C}=\mathrm{O}), 52.50\left(\mathrm{OCH}_{3}\right), 36.09$ $\left(\mathrm{N}-\mathrm{C}\right.$, pyrazole- $\left.\mathrm{CH}_{3}\right), 19.32\left(\mathrm{CH}_{2}-\mathrm{C} \equiv \mathrm{CH}\right), 11.15$ (pyrazole- $\left.\mathrm{CH}_{3}\right), 9.05$ (pyrazole- $\left.\mathrm{CH}_{3}\right)$. HRMS calcd. for $\mathrm{C}_{23} \mathrm{H}_{24} \mathrm{~F}_{6} \mathrm{~N}_{4} \mathrm{O}_{4}\left([\mathrm{M}+\mathrm{H}]^{+}\right)$, 535.1775; found, 535.1768.

2-Allyl-N $N^{1}$-(4-(1,1,1,3,3,3-hexafluoro-2-methoxypropan-2-yl)-2-methoxyphenyl)- $N^{3}-(1,3,5$-trimethyl-1Hpyrazol-4-yl)malonamide (8o): White solid; m.p. $190-191{ }^{\circ} \mathrm{C}$; yield $55.2 \%$; ${ }^{1} \mathrm{H}-\mathrm{NMR}(300 \mathrm{MHz}$, Chloroform- $d$ ): $\delta 9.45(\mathrm{~s}, 1 \mathrm{H}$, pyrazole-NH), $8.40(\mathrm{~d}, J=8.6 \mathrm{~Hz}, 1 \mathrm{H}, \mathrm{Ar}-\mathrm{H}), 8.34(\mathrm{~s}, 1 \mathrm{H}, \mathrm{Ar}-\mathrm{NH}), 7.15$ $(\mathrm{d}, J=8.6 \mathrm{~Hz}, 1 \mathrm{H}, \mathrm{Ar}-\mathrm{H}), 7.05(\mathrm{~s}, 1 \mathrm{H}, \mathrm{Ar}-\mathrm{H}), 5.91-5.77\left(\mathrm{~m}, 1 \mathrm{H}, \mathrm{CH}=\mathrm{CH}_{2}\right), 5.25-5.08\left(\mathrm{~m}, 2 \mathrm{H}, \mathrm{CH}=\mathrm{CH}_{2}\right)$, $3.90\left(\mathrm{~s}, 3 \mathrm{H}, \mathrm{Ar}-\mathrm{OCH}_{3}\right), 3.66\left(\mathrm{~s}, 3 \mathrm{H}, \mathrm{N}-\mathrm{CH}_{3}\right), 3.50(\mathrm{t}, J=7.5 \mathrm{~Hz}, 1 \mathrm{H}, \mathrm{O}=\mathrm{C}-\mathrm{CH}-\mathrm{C}=\mathrm{O}), 3.48(\mathrm{~s}, 3 \mathrm{H}$, $\left.\mathrm{OCH}_{3}\right), 2.84-2.76\left(\mathrm{~m}, 2 \mathrm{H}, \mathrm{O}=\mathrm{C}-\mathrm{CH}-\mathrm{CH}_{2}\right), 2.09\left(\mathrm{~d}, \mathrm{~J}=2.7 \mathrm{~Hz}, 6 \mathrm{H}\right.$, pyrazole- $\left.\mathrm{CH}_{3}\right) .{ }^{13} \mathrm{C}-\mathrm{NMR}(75 \mathrm{MHz}$, Chloroform- $d)$ : $\delta 169.48(\mathrm{C}=\mathrm{O}), 168.11(\mathrm{C}=\mathrm{O}), 148.29\left(\right.$ Ar- $\left.\mathrm{C}_{2}\right), 142.71\left(\mathrm{C}=\mathrm{N}\right.$, pyrazole- $\left.\mathrm{C}_{3}\right), 134.55(\mathrm{C}-\mathrm{N}$, Ar- $\left.\mathrm{C}_{1}\right), 133.23\left(\underline{\mathrm{CH}}=\mathrm{CH}_{2}\right), 128.67\left(\mathrm{C}-\mathrm{N}-\mathrm{N}\right.$, pyrazole- $\left.\mathrm{C}_{5}\right), 123.00\left(\mathrm{Ar}-\mathrm{C}_{4}\right), 122.03\left(\mathrm{q}, J_{\mathrm{CF}}=288.8 \mathrm{~Hz}\right.$, $\left.\mathrm{CF}_{3}\right), 120.74\left(\mathrm{Ar}-\mathrm{C}_{5}\right), 119.40\left(\mathrm{Ar}-\mathrm{C}_{6}\right), 118.05\left(\mathrm{CH}=\mathrm{CH}_{2}\right), 113.72\left(\mathrm{C}-\mathrm{N}\right.$, pyrazole- $\left.\mathrm{C}_{4}\right), 109.50\left(\mathrm{Ar}-\mathrm{C}_{3}\right)$, 82.44 (hept, $\left.J_{\mathrm{CF}}=28.5 \mathrm{~Hz}, \underline{\mathrm{C}}\left(\mathrm{CF}_{3}\right)_{2}\right), 55.61\left(\mathrm{Ar}-\mathrm{OCH}_{3}\right), 55.14(\mathrm{O}=\mathrm{C}-\mathrm{C}-\mathrm{C}=\mathrm{O}), 53.88\left(\mathrm{OCH}_{3}\right), 36.74$ (N-C, pyrazole- $\left.\mathrm{CH}_{3}\right), 35.83\left(\mathrm{O}=\mathrm{C}-\mathrm{CH}-\mathrm{CH}_{2}\right), 10.85$ (pyrazole- $\left.\mathrm{CH}_{3}\right), 9.10$ (pyrazole- $\left.\mathrm{CH}_{3}\right)$. HRMS calcd. for $\mathrm{C}_{23} \mathrm{H}_{26} \mathrm{~F}_{6} \mathrm{~N}_{4} \mathrm{O}_{4}\left([\mathrm{M}+\mathrm{H}]^{+}\right)$, 537.1931; found, 537.1922.

$N^{1}$-(4-(1,1,1,3,3,3-Hexafluoro-2-methoxypropan-2-yl)-3-isopropylphenyl)-2-isopropyl- $N^{3}$-(1,3,5-trimethyl-1Hpyrazol-4-yl)malonamide (8p): White solid; m.p. 86-88 ${ }^{\circ} \mathrm{C}$; yield $51.7 \% ;{ }^{1} \mathrm{H}-\mathrm{NMR}(300 \mathrm{MHz}$, Chloroform-d): $\delta 9.46(\mathrm{~s}, 1 \mathrm{H}$, pyrazole-NH), 8.39 (d, J = 1.5 Hz, 1H, Ar-H), 8.06 (s, 1H, Ar-NH), 7.41 $(\mathrm{d}, J=8.4 \mathrm{~Hz}, 1 \mathrm{H}, \mathrm{Ar}-\mathrm{H}), 7.08(\mathrm{dd}, J=8.5,1.8 \mathrm{~Hz}, 1 \mathrm{H}, \mathrm{Ar}-\mathrm{H}), 3.68\left(\mathrm{~s}, 3 \mathrm{H}, \mathrm{N}-\mathrm{CH}_{3}\right), 3.56\left(\mathrm{~s}, 3 \mathrm{H}, \mathrm{OCH}_{3}\right)$, $2.97(\mathrm{~d}, J=10.4 \mathrm{~Hz}, 1 \mathrm{H}, \mathrm{O}=\mathrm{C}-\mathrm{CH}-\mathrm{C}=\mathrm{O}), 2.95-2.86\left(\mathrm{~m}, 1 \mathrm{H}, \mathrm{Ar}-\mathrm{CH}\left(\mathrm{CH}_{3}\right)_{2}\right), 2.53-2.38\left(\mathrm{~m}, 1 \mathrm{H}, \mathrm{CH}\left(\mathrm{CH}_{3}\right)_{2}\right)$, $2.09\left(\mathrm{~d}, J=5.2 \mathrm{~Hz}, 6 \mathrm{H}\right.$, pyrazole- $\left.\mathrm{CH}_{3}\right), 1.26\left(\mathrm{~d}, J=6.9 \mathrm{~Hz}, 6 \mathrm{H}, \mathrm{Ar}-\mathrm{CH}\left(\mathrm{CH}_{3}\right)_{2}\right), 1.12(\mathrm{dd}, J=17.5,6.6 \mathrm{~Hz}$, 6H, $\left.\mathrm{CH}\left(\mathrm{CH}_{3}\right)_{2}\right) .{ }^{13} \mathrm{C}-\mathrm{NMR}\left(75 \mathrm{MHz}\right.$, Chloroform-d): $\delta 168.66(\mathrm{C}=\mathrm{O}), 168.48(\mathrm{C}=\mathrm{O}), 151.92\left(\mathrm{Ar}-\mathrm{C}_{3}\right)$, $142.66\left(\mathrm{C}=\mathrm{N}\right.$, pyrazole- $\left.\mathrm{C}_{3}\right), 137.24\left(\mathrm{C}-\mathrm{N}-\mathrm{N}\right.$, pyrazole- $\left.\mathrm{C}_{5}\right), 134.50\left(\mathrm{C}-\mathrm{N}, \mathrm{Ar}-\mathrm{C}_{1}\right), 129.37\left(\right.$ Ar- $\left._{4}\right), 122.40$ $\left(\right.$ Ar- $\left.\mathrm{C}_{5}\right), 122.04\left(\mathrm{q}, J_{\mathrm{CF}}=288.8 \mathrm{~Hz}, \mathrm{CF}_{3}\right), 121.35\left(\mathrm{Ar}-\mathrm{C}_{6}\right), 113.80(\mathrm{C}-\mathrm{N}$, pyrazole-C 4$), 112.11\left(\mathrm{Ar}-\mathrm{C}_{2}\right), 84.49$ (hept, $\left.J_{\mathrm{CF}}=28.5 \mathrm{~Hz}, \underline{\mathrm{C}}\left(\mathrm{CF}_{3}\right)_{2}\right), 65.18(\mathrm{O}=\mathrm{C}-\mathrm{C}-\mathrm{C}=\mathrm{O}), 54.28\left(\mathrm{OCH}_{3}\right), 35.89\left(\mathrm{~N}-\mathrm{C}\right.$, pyrazole- $\left.\mathrm{CH}_{3}\right), 33.50$ $\left(\mathrm{Ar}-\mathrm{CH}\left(\mathrm{CH}_{3}\right)_{2}\right), 31.66\left(\mathrm{CH}\left(\mathrm{CH}_{3}\right)_{2}\right), 23.08\left(\mathrm{Ar}-\mathrm{CH}\left(\mathrm{CH}_{3}\right)_{2}\right), 20.36\left(\mathrm{CH}\left(\mathrm{CH}_{3}\right)_{2}\right), 20.28\left(\mathrm{CH}\left(\mathrm{CH}_{3}\right)_{2}\right), 10.80$ (pyrazole- $\mathrm{CH}_{3}$ ), 9.12 (pyrazole- $\mathrm{CH}_{3}$ ). HRMS calcd. for $\mathrm{C}_{25} \mathrm{H}_{32} \mathrm{~F}_{6} \mathrm{~N}_{4} \mathrm{O}_{3}\left([\mathrm{M}+\mathrm{H}]^{+}\right.$), 551.2451; found, 551.2443.

$N^{1}-(4-(1,1,1,3,3,3-H e x a f l u o r o-2-m e t h o x y p r o p a n-2-y l)-3-i s o p r o p y l p h e n y l)-2-i s o b u t y l-N^{3}-(1,3,5-$ trimethyl-1Hpyrazol-4-yl)malonamide (8q): White solid; m.p. $67-69{ }^{\circ} \mathrm{C}$; yield $48.8 \%$; ${ }^{1} \mathrm{H}-\mathrm{NMR}(300 \mathrm{MHz}$, Chloroform-d): $\delta 9.41(\mathrm{~s}, 1 \mathrm{H}$, pyrazole-NH), $8.33(\mathrm{~d}, J=1.8 \mathrm{~Hz}, 1 \mathrm{H}, \mathrm{Ar}-\mathrm{H}), 8.02(\mathrm{~s}, 1 \mathrm{H}, \mathrm{Ar}-\mathrm{NH}), 7.39$ $(\mathrm{d}, J=8.4 \mathrm{~Hz}, 1 \mathrm{H}, \mathrm{Ar}-\mathrm{H}), 7.06(\mathrm{dd}, J=8.5,1.8 \mathrm{~Hz}, 1 \mathrm{H}, \mathrm{Ar}-\mathrm{H}), 3.66\left(\mathrm{~s}, 3 \mathrm{H}, \mathrm{N}-\mathrm{CH}_{3}\right), 3.54\left(\mathrm{~s}, 3 \mathrm{H}, \mathrm{OCH}_{3}\right)$, $3.45(\mathrm{t}, J=7.6 \mathrm{~Hz}, 1 \mathrm{H}, \mathrm{O}=\mathrm{C}-\mathrm{CH}-\mathrm{C}=\mathrm{O}), 2.94-2.85\left(\mathrm{~m}, 1 \mathrm{H}, \mathrm{Ar}-\mathrm{CH}\left(\mathrm{CH}_{3}\right)_{2}\right), 2.06(\mathrm{~d}, J=4.7 \mathrm{~Hz}, 6 \mathrm{H}$, pyrazole- $\left.\mathrm{CH}_{3}\right), 2.00-1.83\left(\mathrm{~m}, 2 \mathrm{H}, \mathrm{O}=\mathrm{C}-\mathrm{CH}-\mathrm{CH}_{2}\right), 1.77-1.68\left(\mathrm{~m}, 1 \mathrm{H}, \mathrm{CH}\left(\mathrm{CH}_{3}\right)_{2}\right), 1.24(\mathrm{~d}, J=6.9 \mathrm{~Hz}, 6 \mathrm{H}$, $\left.\mathrm{Ar}-\mathrm{CH}\left(\mathrm{CH}_{3}\right)_{2}\right), 0.99\left(\mathrm{dd}, J=6.5,4.0 \mathrm{~Hz}, 6 \mathrm{H}, \mathrm{CH}\left(\mathrm{CH}_{3}\right)_{2}\right) .{ }^{13} \mathrm{C}-\mathrm{NMR}(75 \mathrm{MHz}$, Chloroform-d): $\delta 169.08$ $(\mathrm{C}=\mathrm{O}), 169.02(\mathrm{C}=\mathrm{O}), 151.90\left(\mathrm{Ar}^{-\mathrm{C}_{3}}\right), 142.67\left(\mathrm{C}=\mathrm{N}\right.$, pyrazole- $\left.\mathrm{C}_{3}\right), 137.17\left(\mathrm{C}-\mathrm{N}-\mathrm{N}\right.$, pyrazole- $\left.\mathrm{C}_{5}\right), 134.54$ $\left(\mathrm{C}-\mathrm{N}, \mathrm{Ar}-\mathrm{C}_{1}\right), 129.36\left(\mathrm{Ar}-\mathrm{C}_{4}\right), 122.46\left(\mathrm{Ar}^{-\mathrm{C}_{5}}\right), 122.09\left(\mathrm{q}, \mathrm{J}_{\mathrm{CF}}=288.8 \mathrm{~Hz}, \mathrm{CF}_{3}\right), 121.57\left(\mathrm{Ar}-\mathrm{C}_{6}\right), 113.79(\mathrm{C}-\mathrm{N}$, pyrazole- $\left.\mathrm{C}_{4}\right), 112.21\left(\mathrm{Ar}-\mathrm{C}_{2}\right), 84.49$ (hept, $\left.J_{\mathrm{CF}}=28.5 \mathrm{~Hz}, \underline{\mathrm{C}}\left(\mathrm{CF}_{3}\right)_{2}\right), 55.20\left(\mathrm{OCH}_{3}\right), 54.27(\mathrm{O}=\mathrm{C}-\mathrm{C}-\mathrm{C}=\mathrm{O})$, $40.72\left(\mathrm{O}=\mathrm{C}-\mathrm{CH}-\mathrm{CH}_{2}\right), 35.87\left(\mathrm{~N}-\mathrm{C}\right.$, pyrazole- $\left.\mathrm{CH}_{3}\right), 33.50\left(\mathrm{Ar}-\underline{\mathrm{CH}}\left(\mathrm{CH}_{3}\right)_{2}\right), 26.04\left(\underline{\mathrm{CH}}\left(\mathrm{CH}_{3}\right)_{2}\right), 23.07$ 
(Ar- $\left.\mathrm{CH}\left(\mathrm{CH}_{3}\right)_{2}\right), 22.10\left(\mathrm{CH}\left(\mathrm{CH}_{3}\right)_{2}\right), 21.81\left(\mathrm{CH}\left(\mathrm{CH}_{3}\right)_{2}\right), 10.70$ (pyrazole- $\left.\mathrm{CH}_{3}\right), 9.05$ (pyrazole- $\left.\mathrm{CH}_{3}\right)$. HRMS calcd. for $\mathrm{C}_{26} \mathrm{H}_{34} \mathrm{~F}_{6} \mathrm{~N}_{4} \mathrm{O}_{3}\left([\mathrm{M}+\mathrm{H}]^{+}\right)$, 565.2608; found, 565.2601.

$N^{1}$-(4-(1,1,1,3,3,3-Hexafluoro-2-methoxypropan-2-yl)-3-isopropylphenyl)-2-isopentyl- $N^{3}$-(1,3,5-trimethyl-1Hpyrazol-4-yl)malonamide (8r): White solid; m.p. 63-64 ${ }^{\circ} \mathrm{C}$; yield $54.8 \% ;{ }^{1} \mathrm{H}-\mathrm{NMR}(300 \mathrm{MHz}$, Chloroform-d): $\delta 9.40(\mathrm{~s}, 1 \mathrm{H}$, pyrazole-NH), $8.33(\mathrm{~d}, J=1.7 \mathrm{~Hz}, 1 \mathrm{H}, \mathrm{Ar}-\mathrm{H}), 7.97(\mathrm{~s}, 1 \mathrm{H}, \mathrm{Ar}-\mathrm{NH})$, $7.39(\mathrm{~d}, J=8.4 \mathrm{~Hz}, 1 \mathrm{H}, \mathrm{Ar}-\mathrm{H}), 7.07(\mathrm{dd}, J=8.5,1.9 \mathrm{~Hz}, 1 \mathrm{H}, \mathrm{Ar}-\mathrm{H}), 3.66\left(\mathrm{~s}, 3 \mathrm{H}, \mathrm{N}-\mathrm{CH}_{3}\right), 3.54(\mathrm{~s}, 3 \mathrm{H}$, $\left.\mathrm{OCH}_{3}\right), 3.27(\mathrm{t}, J=7.5 \mathrm{~Hz}, 1 \mathrm{H}, \mathrm{O}=\mathrm{C}-\mathrm{CH}-\mathrm{C}=\mathrm{O}), 2.98-2.84\left(\mathrm{~m}, 1 \mathrm{H}, \mathrm{Ar}-\mathrm{CH}\left(\mathrm{CH}_{3}\right)_{2}\right), 2.07(\mathrm{~d}, J=4.4 \mathrm{~Hz}$, $6 \mathrm{H}$, pyrazole- $\left.\mathrm{CH}_{3}\right), 2.05-1.97\left(\mathrm{~m}, 2 \mathrm{H}, \mathrm{O}=\mathrm{C}-\mathrm{CH}-\mathrm{CH}_{2}\right), 1.68-1.55\left(\mathrm{~m}, 1 \mathrm{H}, \mathrm{CH}\left(\mathrm{CH}_{3}\right)_{2}\right), 1.37-1.29$ $\left(\mathrm{m}, 2 \mathrm{H}, \mathrm{CH}_{2}-\mathrm{CH}\left(\mathrm{CH}_{3}\right)_{2}\right), 1.25\left(\mathrm{~d}, J=6.9 \mathrm{~Hz}, 6 \mathrm{H}, \mathrm{Ar}-\mathrm{CH}\left(\mathrm{CH}_{3}\right)_{2}\right), 0.90\left(\mathrm{~d}, J=6.6 \mathrm{~Hz}, 6 \mathrm{H}, \mathrm{CH}\left(\mathrm{CH}_{3}\right)_{2}\right)$. ${ }^{13} \mathrm{C}-\mathrm{NMR}\left(75 \mathrm{MHz}\right.$, Chloroform-d): $\delta 169.04(\mathrm{C}=\mathrm{O}), 168.96(\mathrm{C}=\mathrm{O}), 151.92\left(\mathrm{Ar}-\mathrm{C}_{3}\right), 142.68(\mathrm{C}=\mathrm{N}$, pyrazole- $\left.\mathrm{C}_{3}\right), 137.15\left(\mathrm{C}-\mathrm{N}-\mathrm{N}\right.$, pyrazole- $\left.\mathrm{C}_{5}\right), 134.56\left(\mathrm{C}-\mathrm{N}, \mathrm{Ar}-\mathrm{C}_{1}\right), 129.36\left(\mathrm{Ar}-\mathrm{C}_{4}\right), 122.49\left(\mathrm{Ar}-\mathrm{C}_{5}\right)$, $122.09\left(\mathrm{q}, J_{\mathrm{CF}}=288.8 \mathrm{~Hz}, \mathrm{CF}_{3}\right), 121.66\left(\mathrm{Ar}-\mathrm{C}_{6}\right), 113.77\left(\mathrm{C}-\mathrm{N}\right.$, pyrazole- $\left.\mathrm{C}_{4}\right), 112.25\left(\mathrm{Ar}-\mathrm{C}_{2}\right), 84.49$ (hept, $\left.J_{\mathrm{CF}}=28.5 \mathrm{~Hz}, \underline{\mathrm{C}}\left(\mathrm{CF}_{3}\right)_{2}\right), 57.03(\mathrm{O}=\mathrm{C}-\underline{\mathrm{C}}-\mathrm{C}=\mathrm{O}), 54.27\left(\mathrm{OCH}_{3}\right), 36.24\left(\mathrm{~N}-\mathrm{C}\right.$, pyrazole- $\left.\mathrm{CH}_{3}\right)$, $35.88\left(\mathrm{CH}_{2}-\mathrm{CH}\left(\mathrm{CH}_{3}\right)_{2}\right), 33.50\left(\mathrm{Ar}-\underline{\mathrm{CH}}\left(\mathrm{CH}_{3}\right)_{2}\right), 29.94\left(\mathrm{CH}_{2}-\underline{\mathrm{CH}}\left(\mathrm{CH}_{3}\right)_{2}\right), 27.47\left(\mathrm{O}=\mathrm{C}-\mathrm{CH}-\mathrm{CH}_{2}\right)$, $23.08\left(\mathrm{Ar}-\mathrm{CH}\left(\mathrm{CH}_{3}\right)_{2}\right), 22.06\left(\mathrm{CH}_{2}-\mathrm{CH}\left(\mathrm{CH}_{3}\right)_{2}\right), 21.88\left(\mathrm{CH}_{2}-\mathrm{CH}\left(\mathrm{CH}_{3}\right)_{2}\right), 10.74$ (pyrazole- $\left.\mathrm{CH}_{3}\right)$, 9.07 (pyrazole- $\left.\mathrm{CH}_{3}\right)$. HRMS calcd. for $\mathrm{C}_{27} \mathrm{H}_{36} \mathrm{~F}_{6} \mathrm{~N}_{4} \mathrm{O}_{3}\left([\mathrm{M}+\mathrm{H}]^{+}\right)$, 579.2764; found, 579.2756.

$N^{1}$-(4-(1,1,1,3,3,3-Hexafluoro-2-methoxypropan-2-yl)-3-isopropylphenyl)-2-(prop-2-yn-1-yl)-N3-(1,3,5-trimethyl1H-pyrazol-4-yl)malonamide (8s): White solid; m.p. 69-71 ${ }^{\circ} \mathrm{C}$; yield $56.5 \%$; ${ }^{1} \mathrm{H}-\mathrm{NMR}(300 \mathrm{MHz}$, Chloroform-d): $\delta 9.44$ (s, 1H, pyrazole-NH), 8.27 (d, J = 1.7 Hz, 1H, Ar-H), 7.93 (s, 1H, Ar-NH), 7.39 $(\mathrm{d}, J=8.4 \mathrm{~Hz}, 1 \mathrm{H}, \mathrm{Ar}-\mathrm{H}), 7.08(\mathrm{dd}, J=8.5,1.9 \mathrm{~Hz}, 1 \mathrm{H}, \mathrm{Ar}-\mathrm{H}), 3.66\left(\mathrm{~s}, 3 \mathrm{H}, \mathrm{N}-\mathrm{CH}_{3}\right), 3.56-3.51(\mathrm{~m}, 4 \mathrm{H}$, $\mathrm{O}=\mathrm{C}-\mathrm{CH}-\mathrm{C}=\mathrm{O}$ and $\left.\mathrm{O}-\mathrm{CH}_{3}\right), 2.98-2.93\left(\mathrm{~m}, 2 \mathrm{H}, \mathrm{CH}_{2}-\mathrm{C} \equiv \mathrm{CH}\right), 2.93-2.86\left(\mathrm{~m}, 1 \mathrm{H}, \mathrm{Ar}-\mathrm{CH}\left(\mathrm{CH}_{3}\right)_{2}\right), 2.12$ $(\mathrm{t}, J=2.6 \mathrm{~Hz}, 1 \mathrm{H}, \mathrm{C} \equiv \mathrm{CH}), 2.08\left(\mathrm{~d}, J=6.1 \mathrm{~Hz}, 6 \mathrm{H}\right.$, pyrazole- $\left.\mathrm{CH}_{3}\right), 1.25\left(\mathrm{~d}, J=6.9 \mathrm{~Hz}, 6 \mathrm{H}, \mathrm{Ar}-\mathrm{CH}\left(\mathrm{CH}_{3}\right)_{2}\right)$. ${ }^{13} \mathrm{C}-\mathrm{NMR}\left(75 \mathrm{MHz}\right.$, Chloroform-d): $\delta 167.52(\mathrm{C}=\mathrm{O}), 166.65(\mathrm{C}=\mathrm{O}), 151.97\left(\mathrm{Ar}-\mathrm{C}_{3}\right), 142.79(\mathrm{C}=\mathrm{N}$, pyrazole- $\left.\mathrm{C}_{3}\right), 136.79\left(\mathrm{C}-\mathrm{N}-\mathrm{N}\right.$, pyrazole- $\left.\mathrm{C}_{5}\right), 134.83\left(\mathrm{C}-\mathrm{N}, \mathrm{Ar}-\mathrm{C}_{1}\right), 129.37\left(\mathrm{Ar}_{-} \mathrm{C}_{4}\right), 122.78\left(\mathrm{Ar}-\mathrm{C}_{5}\right)$, $122.14\left(\mathrm{Ar}-\mathrm{C}_{6}\right), 122.09\left(\mathrm{q}, J_{\mathrm{CF}}=288.8 \mathrm{~Hz}, \mathrm{CF}_{3}\right), 113.54\left(\mathrm{C}-\mathrm{N}\right.$, pyrazole- $\left.\mathrm{C}_{4}\right), 112.65\left(\mathrm{Ar}-\mathrm{C}_{2}\right), 84.43$ (hept, $\left.J_{\mathrm{CF}}=28.5 \mathrm{~Hz}, \underline{\mathrm{C}}\left(\mathrm{CF}_{3}\right)_{2}\right), 79.52(\underline{\mathrm{C}} \equiv \mathrm{CH}), 71.27(\mathrm{C} \equiv \underline{\mathrm{CH}}), 54.86(\mathrm{O}=\mathrm{C}-\underline{\mathrm{C}}-\mathrm{C}=\mathrm{O}), 54.30\left(\mathrm{OCH}_{3}\right), 35.87(\mathrm{~N}-\mathrm{C}$, pyrazole- $\left.\mathrm{CH}_{3}\right), 33.47\left(\mathrm{Ar}-\mathrm{CH}\left(\mathrm{CH}_{3}\right)_{2}\right), 23.07\left(\mathrm{Ar}-\mathrm{CH}\left(\mathrm{CH}_{3}\right)_{2}\right), 20.88\left(\mathrm{CH}_{2}-\mathrm{C} \equiv \mathrm{CH}\right), 10.71$ (pyrazole- $\left.\mathrm{CH}_{3}\right)$, 9.05 (pyrazole- $\left.\mathrm{CH}_{3}\right)$. HRMS calcd. for $\mathrm{C}_{25} \mathrm{H}_{28} \mathrm{~F}_{6} \mathrm{~N}_{4} \mathrm{O}_{3}\left([\mathrm{M}+\mathrm{H}]^{+}\right)$, 547.2138; found, 547.2131.

2-Allyl-N $N_{-1}$-4-(1,1,1,3,3,3-hexafluoro-2-methoxypropan-2-yl)-3-isopropylphenyl)- $N^{3}-(1,3,5-$ trimethyl-1H-pyrazol4-yl)malonamide (8t): White solid; m.p. $58-60{ }^{\circ} \mathrm{C}$; yield 56.8\%; ${ }^{1} \mathrm{H}-\mathrm{NMR}(300 \mathrm{MHz}$, Chloroform- $d$ ): $\delta 9.39(\mathrm{~s}, 1 \mathrm{H}$, pyrazole-NH), $8.28(\mathrm{~d}, J=1.7 \mathrm{~Hz}, 1 \mathrm{H}, \mathrm{Ar}-\mathrm{H}), 7.90(\mathrm{~s}, 1 \mathrm{H}, \mathrm{Ar}-\mathrm{NH}), 7.39(\mathrm{~d}, J=8.5 \mathrm{~Hz}, 1 \mathrm{H}$, Ar-H), 7.08 (dd, J = 8.5, $1.9 \mathrm{~Hz}, 1 \mathrm{H}, \mathrm{Ar}-\mathrm{H}), 5.92-5.78\left(\mathrm{~m}, 1 \mathrm{H}, \mathrm{CH}=\mathrm{CH}_{2}\right), 5.29-5.10\left(\mathrm{~m}, 2 \mathrm{H}, \mathrm{CH}=\mathrm{CH}_{2}\right)$, $3.66\left(\mathrm{~s}, 3 \mathrm{H}, \mathrm{N}-\mathrm{CH}_{3}\right), 3.54\left(\mathrm{~s}, 3 \mathrm{H}, \mathrm{O}-\mathrm{CH}_{3}\right), 3.38(\mathrm{t}, J=7.5 \mathrm{~Hz}, 1 \mathrm{H}, \mathrm{O}=\mathrm{C}-\mathrm{CH}-\mathrm{C}=\mathrm{O}), 2.96-2.87(\mathrm{~m}, 1 \mathrm{H}$, Ar- $\left.\mathrm{CH}\left(\mathrm{CH}_{3}\right)_{2}\right), 2.80\left(\mathrm{t}, J=7.2 \mathrm{~Hz}, 2 \mathrm{H}, \mathrm{O}=\mathrm{C}-\mathrm{CH}-\mathrm{CH}_{2}\right), 2.06\left(\mathrm{~d}, J=4.9 \mathrm{~Hz}, 6 \mathrm{H}\right.$, pyrazole- $\left.\mathrm{CH}_{3}\right), 1.25$ $\left(\mathrm{d}, J=6.9 \mathrm{~Hz}, 6 \mathrm{H}, \mathrm{Ar}-\mathrm{CH}\left(\mathrm{CH}_{3}\right)_{2}\right) .{ }^{13} \mathrm{C}-\mathrm{NMR}(75 \mathrm{MHz}$, Chloroform- $d): \delta 168.30(\mathrm{C}=\mathrm{O}), 168.14(\mathrm{C}=\mathrm{O})$, $151.95\left(\right.$ Ar- $\left._{3}\right), 142.71\left(\mathrm{C}=\mathrm{N}\right.$, pyrazole- $\left.\mathrm{C}_{3}\right), 136.97\left(\mathrm{C}-\mathrm{N}-\mathrm{N}\right.$, pyrazole- $\left.\mathrm{C}_{5}\right), 134.66\left(\mathrm{C}-\mathrm{N}, \mathrm{Ar} \mathrm{C}_{1}\right), 133.18$ $\left(\underline{\mathrm{CH}}=\mathrm{CH}_{2}\right), 129.37\left(\mathrm{Ar}-\mathrm{C}_{4}\right), 122.09\left(\mathrm{q}, J_{\mathrm{CF}}=288.8 \mathrm{~Hz}, \mathrm{CF}_{3}\right), 122.66\left(\mathrm{Ar}-\mathrm{C}_{5}\right), 121.87\left(\mathrm{Ar}-\mathrm{C}_{6}\right), 118.12$ $\left(\mathrm{CH}=\mathrm{CH}_{2}\right), 113.60\left(\mathrm{C}-\mathrm{N}\right.$, pyrazole- $\left.\mathrm{C}_{4}\right), 112.42\left(\mathrm{Ar}-\mathrm{C}_{2}\right), 84.47$ (hept, $\left.J_{\mathrm{CF}}=28.5 \mathrm{~Hz}, \underline{\mathrm{C}}\left(\mathrm{CF}_{3}\right)_{2}\right), 56.32$ $(\mathrm{O}=\mathrm{C}-\underline{\mathrm{C}}-\mathrm{C}=\mathrm{O}), 54.28\left(\mathrm{OCH}_{3}\right), 35.89\left(\mathrm{~N}-\mathrm{C}\right.$, pyrazole- $\left.\mathrm{CH}_{3}\right), 35.86\left(\mathrm{O}=\mathrm{C}-\mathrm{CH}-\underline{-} \mathrm{CH}_{2}\right), 33.48\left(\mathrm{Ar}-\underline{\mathrm{C}} \mathrm{H}\left(\mathrm{CH}_{3}\right)_{2}\right)$, $23.09\left(\mathrm{Ar}-\mathrm{CH}\left(\mathrm{CH}_{3}\right)_{2}\right), 10.72$ (pyrazole- $\left.\mathrm{CH}_{3}\right), 9.08$ (pyrazole- $\left.\mathrm{CH}_{3}\right)$. HRMS calcd. for $\mathrm{C}_{25} \mathrm{H}_{30} \mathrm{~F}_{6} \mathrm{~N}_{4} \mathrm{O}_{3}$ $\left([\mathrm{M}+\mathrm{H}]^{+}\right), 549.2295$; found, 549.2287 .

\subsection{Bioassay Methods}

All of the biological assays were performed on representative test organisms reared in the laboratory. Each bioassay was repeated at $25 \pm 1{ }^{\circ} \mathrm{C}$ according to statistical requirements. All compounds were dissolved in dimethyl sulfoxide and diluted with distilled water containing Triton X-100 to obtain a series of concentrations. Control check (CK) was treated as the same without compounds. Assessments were based on a dead/alive scale, and mortality rates were rectified using Abbott's formula [27]. Evaluations of the activities of the compounds were based on a percentage 
scale of 0 to 100, where 0 equals no activity, and 100 equals total kill. The error of the experiments was kept within $5.0 \%$. Given the lack of appropriate methods and purchasing channels to obtain the pyflubumide standard in our present work, commercial insecticides, such as fenpyroximate, flubendiamide, and imidacloprid, were selected as positive controls under the same conditions.

\subsubsection{Acaricidal Activity Against Tetranychus Cinnabarinus}

The acaricidal activities of the target compounds and fenpyroximate against Tetranychus cinnabarinus were evaluated using the reported procedure [28]. Sieva bean plants (Phaseolus vulgaris) with primary leaves expanded to $10 \mathrm{~cm}$ in size were selected and cut back to one plant per pot. A small piece was cut from a leaf taken from the main colony and placed on each leaf of the test plants. This was done approximately $2 \mathrm{~h}$ before treatment to allow the mites to move to the test plant and lay eggs. The size of the leaf piece from the main colony was varied to obtain approximately 30 mites per leaf. At the time of the treatment, the piece of leaf used to transfer the mites was removed and discarded. The mite-infested plants were dipped in the test solution for $3 \mathrm{~s}$ with agitation and set in a fume hood to dry. Plants were kept for $48 \mathrm{~h}$ before the numbers of live and dead adults were counted. Each treatment was repeated with triplicate experiments, and each replicate involved 30 adult mites.

\subsubsection{Insecticidal Activity Against Plutella xylostella}

The insecticidal activities of the target compounds and flubendiamide against Plutella xylostella were evaluated using the reported procedure [29]. Leaf disks $(6 \mathrm{~cm} \times 2 \mathrm{~cm})$ were cut from fresh cabbage leaves and then dipped into the test solution for $5 \mathrm{~s}$. After air-drying, the treated leaf disks were placed individually into glass tubes. Each dried treated leaf disk was infested with 10 third-instar Plutella xylostella larvae. Mortality was assessed $48 \mathrm{~h}$ after treatment. Each treatment was repeated with triplicate experiments and each replicate involved 10 third-instar Plutella xylostella larvae.

\subsubsection{Insecticidal Activity Against Aphis craccivora}

The insecticidal activities of the target compounds and imidacloprid against Aphis craccivora were evaluated using the reported procedure [30]. Leaves from the soybean plant with 20 apterous adults were dipped in the test solution for $5 \mathrm{~s}$ and the excess solution was blotted with filter paper. The mortality rates were evaluated $36 \mathrm{~h}$ after treatment. Each treatment was repeated with triplicate experiments and each replicate involved 20 apterous adults.

\section{Conclusions}

In summary, twenty novel 1,3,5-trimethylpyrazole-containing malonamide derivatives were designed and synthesized. The target compounds were evaluated for acaricidal and insecticidal activities against Tetranychus cinnabarinus, Plutella xylostella, and Aphis craccivora. Preliminary bioassay results indicated that compounds $8 \mathrm{~m}$ and $8 \mathrm{p}$ were found to have moderate activities against Tetranychus cinnabarinus. Compounds $\mathbf{8} \mathbf{i}$ and $\mathbf{8 0}$ exhibited good insecticidal activities against Plutella xylostella. In addition, compounds $\mathbf{8} \mathbf{p}$ and $\mathbf{8} \mathbf{q}$ possessed potent insecticidal activities against Aphis craccivora. Although the target compounds did not exhibit excellent acaricidal activities against Tetranychus cinnabarinus, as we expected, some of the target compounds showed good insecticidal activities against Plutella xylostella and Aphis craccivora, respectively. The preliminary analyses of structure-activity relationships (SAR) indicated that the methoxy-substituted hexafluoroisopropyl group and variations of $Y$ groups in the position of phenyl ring markedly affected the acaricidal and insecticidal activities. In addition, different types of substituents $\left(\mathrm{R}_{2}\right)$ have a great influence on the biological activity of different pest species. Notably, these findings demonstrated that the synthesis of 1,3,5-trimethylpyrazole-containing malonamide derivatives could be considered as a new template for pesticide development. Further structural optimization and bioactivities are in progress. 
Supplementary Materials: The spectra of ${ }^{1} \mathrm{H}-\mathrm{NMR},{ }^{13} \mathrm{C}-\mathrm{NMR}$, and HRMS for all the synthesized compounds are available online.

Author Contributions: Writing-Original Draft Preparation, Q.-B.L.; Writing-Review and Editing, C.-H.R. and S.-Z.L.; Project Administration, Q.-B.L., M.L., Q.L., T.F., and Z.-Y.X.

Funding: This research was funded by the National Key Research and Development Program of China (No. 2017YFD0200504).

Conflicts of Interest: The authors declare no conflict of interest.

\section{References}

1. Xiao, N.W.; Jing, B.; Ge, F.; Liu, X.H. The fate of herbicide acetochlor and its toxicity to Eisenia fetida under laboratory conditions. Chemosphere 2006, 62, 1366-1373. [CrossRef] [PubMed]

2. Abigail, M.E.A.; Samuel, S.M.; Ramalingam, C. Addressing the environmental impacts of butachlor and the available remediation strategies: A systematic review. Int. J. Environ. Sci. Technol. 2015, 12, 4025-4036. [CrossRef]

3. Avenot, H.F.; Michailides, T.J. Resistance to boscalid fungicide in Alternaria alternata isolates from pistachio in California. Plant Dis. 2007, 91, 1345-1350. [CrossRef]

4. Avenot, H.F.; Michailides, T.J. Progress in understanding molecular mechanisms and evolution of resistance to succinate dehydrogenase inhibiting (SDHI) fungicides in phytopathogenic fungi. Crop Prot. 2010, 29, 643-651. [CrossRef]

5. Sharma, A.K.; Singh, D.P.; Kumar, J.; Singh, A.; Tewari, A.N.; Singh, K.P.; Karwasra, S.S.; Grewal, A.S. Efficacy of thifluzamide in the control of loose smut of wheat (Triticum aestivum) caused by Ustilago segetum. Indian J. Agric. Sci. 2001, 71, 648-649.

6. Illicachi, L.A.; Montalvo-Acosta, J.J.; Insuasty, A.; Quiroga, J.; Abonia, R.; Sortino, M.; Zacchino, S.; Insuasty, B. Synthesis and DFT calculations of novel Vanillin-Chalcones and their 3-aryl-5-(4-(2-(dimethylamino)ethoxy)-3-methoxyphenyl)-4,5-dihydro- $1 H$-pyrazole-1-carbaldehyde derivatives as antifungal agents. Molecules 2017, 22, 1476. [CrossRef] [PubMed]

7. Nossier, E.S.; Fahmy, H.H.; Khalifa, N.M.; El-Eraky, W.I.; Baset, M.A. Design and synthesis of novel pyrazole-substituted different nitrogenous heterocyclic ring systems as potential anti-inflammatory agents. Molecules 2017, 22, 512. [CrossRef]

8. Fahmy, H.H.; Khalifa, N.M.; Ismail, M.M.F.; El-Sahrawy, H.M.; Nossier, E.S. Biological validation of novel polysubstituted pyrazole candidates with in vitro anticancer activities. Molecules 2016, 21, 271. [CrossRef]

9. Colliot, F.; Kukorowski, K.A.; Hawkins, D.W.; Roberts, D.A. Fipronil: A new soil and foliar broad spectrum insecticide. In Proceedings of the Brighton Crop Protection Conference, Pests and Diseases, Brighton, UK, 23-26 November 1992.

10. Vicentini, C.B.; Romagnoli, C.; Andreotti, E.; Mares, D. Synthetic pyrazole derivatives as growth inhibitors of some phytopathogenic fungi. J. Agric. Food Chem. 2007, 55, 10331-10338. [CrossRef]

11. Wakabayashi, K.; Boger, P. Target sites for herbicides: Entering the 21st century. Pest Manag. Sci. 2002, 58, 1149-1154. [CrossRef]

12. Lein, W.; Bornke, F.; Reindl, A.; Ehrhardt, T.; Stitt, M.; Sonnewald, U. Target-based discovery of novel herbicides. Curr. Opin. Plant Biol. 2004, 7, 219-225. [CrossRef] [PubMed]

13. Clark, R.D. Synthesis and QSAR of herbicidal 3-pyrazolyl alpha, alpha, alpha-trifluorotolyl ethers. J. Agric. Food Chem. 1996, 44, 3643-3652. [CrossRef]

14. Casida, J.E. Golden age of RyR and GABA-R diamide and isoxazoline insecticides: Common genesis, serendipity, surprises, selectivity, and safety. Chem. Res. Toxicol. 2015, 28, 560-566. [CrossRef] [PubMed]

15. Chen, Q.C.; Xiong, L.X.; Luo, M.; Wang, J.; Hu, C.Y.; Zhang, X.; Yu, S.J.; Li, Y.H.; Sun, D.Q. Synthesis, larvicidal activities and antifungal activities of novel chlorantraniliprole derivatives and their target in the ryanodine receptor. Molecules 2015, 20, 3854-3867. [CrossRef] [PubMed]

16. Furuya, T.; Machiya, K.; Fujioka, S.; Nakano, M.; Inagaki, K. Development of a novel acaricide, pyflubumide. J. Pestic. Sci. 2017, 42, 132-136. [CrossRef] [PubMed]

17. Nakano, M.; Yasokawa, N.; Suwa, A.; Fujioka, S.; Furuya, T.; Sakata, K. Mode of action of novel acaricide pyflubumide: Effects on the mitochondrial respiratory chain. J. Pestic. Sci. 2015, 40, 19-24. [CrossRef] 
18. Tohnishi, M.; Nakao, H.; Furuya, T.; Seo, A.; Kodama, H.; Tsubata, K.; Fujioka, S.; Kodama, H.; Hirooka, T.; Nishimatsu, T. Flubendiamide, a novel insecticide highly active against lepidopterous insect pests. J. Pestic. Sci. 2005, 30, 354-360. [CrossRef]

19. Masaki, T.; Yasokawa, N.; Tohnishi, M.; Nishimatsu, T.; Tsubata, K.; Inoue, K.; Motoba, K.; Hirooka, T. Flubendiamide, a novel $\mathrm{Ca}^{2+}$ channel modulator, reveals evidence for functional cooperation between $\mathrm{Ca}^{2+}$ pumps and $\mathrm{Ca}^{2+}$ release. Mol. Pharmacol. 2006, 69, 1733-1739. [CrossRef] [PubMed]

20. Lahm, G.P.; Selby, T.P.; Freudenberger, J.H.; Stevenson, T.M.; Myers, B.J.; Seburyamo, G.; Smith, B.K.; Flexner, L.; Clark, C.E.; Cordova, D. Insecticidal anthranilic diamides: A new class of potent ryanodine receptor activators. Bioorg. Med. Chem. Lett. 2005, 15, 4898-4906. [CrossRef] [PubMed]

21. Mekonnen, T.F.; Panne, U.; Koch, M. New photodegradation products of the fungicide fluopyram: Structural elucidation and mechanism identification. Molecules 2018, 23, 2940. [CrossRef] [PubMed]

22. Piqueras, C.M.; Latorre, B.A.; Torres, R. Effectiveness of isofetamid, a new succinate dehydrogenase inhibitor fungicide, in the control of grapevine gray mold. Ciencia e Investigación Agraria 2014, 41, 365-374. [CrossRef]

23. Furuya, T.; Suwa, A.; Nakano, M.; Fujioka, S.; Yasokawa, N.; Machiya, K. Synthesis and biological activity of a novel acaricide, pyflubumide. J. Pestic. Sci. 2015, 40, 38-43. [CrossRef]

24. Limanto, J.; Krska, S.W.; Dorner, B.T.; Vazquez, E.; Yoshikawa, N.; Tan, L. Dynamic kinetic resolution: Asymmetric transfer hydrogenation of alpha-alkyl-substituted beta-ketoamides. Org. Lett. 2010, 12, 512-515. [CrossRef] [PubMed]

25. Masanobu, O.; Oona, Y.A.; Eiji, K.; Kenji, T. A Process for Producing Perfluoroalkylaniline Derivatives. EP Patent 1006102 A2, 7 June 2000.

26. Noboru, A.; Osamu, S. Preparation of Aniline Derivatives. JP Patent 2012067060A, 5 April 2012.

27. Abbott, W.S. A method of computing the effectiveness of an insecticide. J. Econ. Entomol. 1925, 18, $265-267$. [CrossRef]

28. Luo, J.X.; Lai, T.; Guo, T.; Chen, F.; Zhang, L.L.; Ding, W.; Zhang, Y.Q. Synthesis and acaricidal activities of scopoletin phenolic ether derivatives: QSAR, molecular docking study and in silico ADME predictions. Molecules 2018, 23, 995. [CrossRef] [PubMed]

29. Wu, J.; Kang, S.H.; Yuan, Q.K.; Luo, L.J.; Ma, J.; Shi, Q.C.; Yang, S. N-substituted 5-chloro-6-phenylpyridazin3(2H)-ones: Synthesis, insecticidal activity against Plutella xylostella (L.) and SAR study. Molecules 2012, 17, 9413-9420. [CrossRef]

30. Liu, X.Q.; Liu, Y.Q.; Shao, X.S.; Xu, Z.P.; Xu, X.Y.; Li, Z. Synthesis and insecticidal evaluation of tetrahydroimidazo 1,2-a pyridin-5(1H)-one derivatives. Chin. Chem. Lett. 2016, 27, 7-10. [CrossRef]

Sample Availability: Samples of the compounds $\mathbf{8 a - 8 t}$ are available from the authors.

(C) 2019 by the authors. Licensee MDPI, Basel, Switzerland. This article is an open access article distributed under the terms and conditions of the Creative Commons Attribution (CC BY) license (http:/ / creativecommons.org/licenses/by/4.0/). 